\title{
A Study of Neural-Network-Based Classifiers for Material Classification
}

\author{
H.K. Lam, Senior Member, IEEE, Udeme Ekong, Hongbin Liu Member, IEEE, Bo \\ Xiao, Hugo Araujo, Sai Ho Ling, Senior Member, IEEE and Kit Yan Chan
}

\begin{abstract}
In this paper, the performance of the commonly used neural-network-based classifiers is investigated on solving a classification problem which aims to identify the object nature based on surface features of the object. When the surface data is obtained, a proposed feature extraction method is used to extract the surface feature of the object. The extracted features are then used as the inputs for the classifier. This research studies eighteen household objects which are requisite to our daily life. Six commonly used neural-network-based classifiers, namely one-against-all, weighted one-against-all, binary coded, parallel-structured, weighted parallel structured and tree-structured, are investigated. The performance for the six neural-network-based classifiers is evaluated based on recognition accuracy for individual object. Also, two traditional classifiers, namely k-nearest neighbor classifier and naive Bayes classifier, are employed for the comparison purposes. To evaluate robustness property of the classifiers, the original clean data is contaminated with Gaussian white noise. Experimental results show that the parallel-structured, tree-structured and the naive Bayes classifiers outperform the others under the noise-free data. The tree-structured classifier demonstrates the best robustness property under the noisy data.
\end{abstract}

\section{Index Terms}

Classifier, Material Classification, Neural Networks.

\section{INTRODUCTION}

This work was partially supported by King's College London, National Natural Science Foundation of China (61304003 and 61203002), Fundação para a Ciência e Tecnologia (grant number SFHR/BD/44162/2008) and European Social Fund in the POPH framework.

H.K. Lam, Udeme Ekong, Hongbin Liu, Bo Xiao and Hugo Araujois are with the Department of Informatics, King's College London, Strand, London, WC2R 2LC, United Kingdom. e-mail: \{hak-keung.lam, udeme.ekong, hongbin.liu, bo.xiao, hugo.araujo\}@kcl.ac.uk.

Sai Ho Ling is with the Centre for Health Technologies, Faculty of Engineering and Information Technology, University of Technology, Sydney, NSW, Australia. e-mail: Steve.Ling@uts.edu.au.

Kit Yan Chan is with the Department of Electrical and Computer Engineering, Curtin University, Perth, Australia. e-mail: kit.chan@curtin.edu.au.

Manuscript received 2013. 
BJECT classification aims to classify an unknown object into a pre-determined group which consists of a set of pre-classified objects with similar features to that unknown object. This is

a very important field of study that has a diverse number of applications such as risk management of investment [1], hand-writing recognition [2] and speech recognition [3].

In the literature, the main methods of classification can be found as logic based method (e.g., decision trees), perceptron based methods (e.g., single layer perceptrons [4] and neural networks [5]), statistical approach (e.g., Bayesian classification [6]), instance-based methods (e.g., nearest neighbor algorithm [7], [8]) and support vector machine (SVM) methods [9].

The decision tree technique is a subset of the logic based classification method, it performs classification by sorting the inputs based on the inherent feature values. The nodes in a decision tree are representative of the feature values [10]. This method has been improving classification accuracy and interpretability of loan granting decisions [1]. The decision tree can improve the classification accuracy of the process, and also it is transparent and can be easily deciphered. Hence, for example, it is attractive for investment bankers who are required by law to give reason for a loan denial. The performance of the decision tree can be further improved by incorporating with neural networks, in order to utilize the distinct nature of processing adopted by both approaches [11]. However, if the splitting rule of the decision tree makes a wrong decision, it is impossible to return to the correct path resulting in an accumulation of errors. Also, an increase in the number of learned rules leads to the training algorithm trying to memorise the training set instead of discovering the rules that governing the patterns of it resulting in poor predictions.

A single layer perceptron [4] introduced by Rosenblatt in 1962 has created revolution in the artificial intelligence field, which has led to a number of perceptron-based techniques. A single layer perceptron can be simply described as a component that computes the sum of weighted inputs which is then fed to the output of the system. The outputs are then compared with the targets where the difference is employed to adjust the weights until the desired level of accuracy is derived. This approach demonstrates a major limitation that the single layer perceptron can only learn linearly separable problems, thus it is incompatible on addressing non-linearity. Despite the limitation, the single layer perceptron has been applied effectively on finger print matching [4] and image detection [12] applications.

Bayesian decision theory [6] is fundamental to statistical classification methods which provide a model for the classification procedures. The Bayesian classifier is based on the assumption that equal 
prior probabilities exists for all classes [13] which help in resolving conflicts that occur when two or more classes are not well separable resulting in improving the classification accuracy. However, the posterior probabilities cannot be determined directly [5]. The Bayesian classifier was applied successfully in weeds identification [6]. Recently, a hybrid Bayesian classifier [13] has been proposed, and the results demonstrated that the classification capability can be improved.

Another classification method is based on the k-nearest neighbour technique (kNN) [7] which is good in dealing with text based problems such as visual category recognition [8]. The basic principle is that objects in a data set generally exists in the neighbourhood of other objects with similar properties. The technique finds the " $\mathrm{k}$ " nearest objects to the particular input and determines its class by looking for the most frequent class label. In this technique, the distance between objects is more important than their individual positions. The main disadvantages of the $\mathrm{kNN}$ technique are the large memory requirements and the lack of a logical way of choosing "k", this would make it difficult in a classification application as different data sets would require different optimized value of " $k$ " in order to improve the performance of this method [10]. Furthermore, the precision accuracy can be reduced when there are too many classes or when an uneven density of training samples is presented. A clustering-based method is proposed in [14] to solve this problem as training data is being pre-processed via a clustering algorithm and then classified with a novel $\mathrm{kNN}$ algorithm that adjusts the value of "k" with each iteration.

Neural networks [5] consist of 3 distinct segments that the input units which have the primary responsibility of receiving information; the hidden units which carry out the processing and the output units which store the processed results [10]. The neural network is first trained on a set of data to determine the input-output mapping. The weights of connections between neurons are then established and the training network can then be used to classify a new set of data. The backpropagation algorithm [5] is a widely used method for training the neural network and improving its accuracy. It is done by calculating the error between the actual and desired output, adjusting the weights accordingly and then repeating the process until an acceptable level of accuracy is achieved. Neural network is non-linear in nature and demonstrates a universal approximation capability [15] which makes it ideal for dealing with complex input-output relationships such as classification problems. One of the classification applications of neural networks has been used in stock market prediction where different classification architectures were applied in the classification of system input (for example, historical stock market price) into "buy", "sell" or "hold" advices for investing in the S\&P 500 [16]. 
Support vector machines (SVMs) [9] are supervised learning methods that can be used for various data mining applications including classification and time-series analysis. The main concept of the SVMs is to obtain a hyperplane to separate two data classes. Mature theory has been developed to determine the optimal hyperplane by maximizing the distance between the hyperplane and the support vectors for reduction of generalization error for both linearly and nonlinearly separable cases. The solutions are unique and consistent and there are less occurrences of overfitting. However, it demonstrates a high algorithmic complexity and results are not transparent [10]. A novel method was proposed in [17] to tackle the problem of high complexity when large data sets are used. A two-phase approach is adopted. In the first phase, clustering techniques are applied to obtain approximate classes for all the input data, In the second phase, fine-tuning of the classified data by using the instances that are in close proximity to the approximated hyperplane obtained from the first phase is then performed.

In this paper, we consider a classification problem in material surface recognition of an unknown object using a contact sensing fingertip, which demonstrates a wide range of potential domestic and industrial applications, such as on robot-assisted surgery [18]-[21], blind grasping application [22], [23], pose classification [24], prosthetic limbs [25], quality assurance [26], shape extraction and industrial inspection [27], [28], and brain-machine-brain interface [29]. The properties of the object surface which are important for the aid of recognition are the frictional coefficients, texture, compliance and roughness. The data is obtained through an active surface exploration [30], [31] with the aid of contact-sensing fingertip which can accurately identify the normal and frictional force of the object. During the experiments, the contact sensing fingertip slides along the object with short strokes whilst increasing/decreasing the velocity as is appropriate. The properties of the vibrations caused by this action are then used as the input data. A feature vector is extracted from the raw data to reduce the number of data points used for the classification procedure. It is of utmost importance that the contact sensing fingertip is able to differentiate between the objects and that is the basis of emphasis and importance for the research being conducted in this paper.

In view of the superior learning and generalization capability of the neural networks, we are motivated to implement classifiers using neural networks to deal with the material classification problem [32][34]. In this study, the characteristics of the neural networks are considered for the implementation of neural-network-based classifiers, demonstrating different levels of flexibility, scalability and complexity. Six neural-networked-based classifiers, namely one-against-all, weighted one-against-all, binary 
coded, parallel structured, weighted parallel structured, tree-structured, are introduced for recognition of materials touched by the robot finger. In order to make a comparison, two traditional classification methods, namely k-nearest neighbor classifier and the naive Bayes classifier, are considered. Their recognition performance is investigated thoroughly using the dataset collected from experiments. To investigate the robustness property of the classifiers, Gaussian white noise is added to the test dataset and the recognition performance is evaluated. By investigating the recognition performance of the introduced classifiers, the most suitable classifier for the material surface classification problem can be recommended.

This paper is arranged as follow: After the introduction, we present the basic principles and theory behind the neural network in Section II. Section III presents the 6 neural network based classifiers and comments on their flexibility, scalability and complexity. The robustness of all the classifiers are also included. Section IV presents and discusses the results produced from the simulations under both the original testing data case and noisy data case. A conclusion is then drawn in Section V.

\section{NeURAL Networks}

In this section, a brief discussion of a fully-connected feed-forward neural networks with one input layer, $n_{l}$ hidden layers and one output layer is considered. The $t^{t h}$ input of the neural network is given by $\mathbf{x}(t)=\left[\begin{array}{lllll}x_{1}(t) & x_{2}(t) & \ldots & x_{n_{\text {in }}}(t)\end{array}\right]$ and the $t^{\text {th }}$ output as $\mathbf{y}(t)=\left[\begin{array}{llll}y_{1}(t) & y_{2}(t) & \ldots & y_{n_{\text {out }}}(t)\end{array}\right]$ where $n_{\text {in }}$ denotes the number of input nodes in the input layer and $n_{\text {out }}$ denotes the number of output nodes in the output layer. The output of the $j$-th node in the input layer is given as follows:

$$
f_{i}^{(0)}(t)=x_{i}(t), i=1,2, \ldots, n_{\text {in }}
$$

and the output of the $j$-th node in the $n_{l}$-th hidden layer is given as follows:

$$
f_{i}^{\left(n_{l}\right)}(t)=t f_{n_{l}}\left(\sum_{j=1}^{n_{n_{h}}^{\left(n_{l}-1\right)}} w_{i j}^{\left(n_{l}\right)} f_{j}^{\left(n_{l}-1\right)}(t)-b_{j}^{\left(n_{l}\right)}\right), i=1,2, \ldots, n_{h}^{\left(n_{l}\right)},
$$

where $t f_{n_{l}}(\cdot)$ denotes the transfer function; $n_{h}^{\left(n_{l}\right)}$ denotes the number of hidden nodes, $b_{i}^{\left(n_{l}\right)}$ denotes the bias in the $n_{l}$-th hidden layer; and $w_{i j}^{\left(n_{l}\right)}$ denotes the weight between the $j$-th node in the $n_{h}^{\left(n_{l}-1\right)}$-th hidden layer and the $i$-th node in the $n_{h}^{\left(n_{l}\right)}$-th hidden layer. 
The output of the neural network is given as follows:

$$
y_{i}(t)=t f_{n_{l}+1}\left(\sum_{j=1}^{n_{n_{h}}^{\left(n_{l}\right)}} w_{i j}^{\left(n_{l}+1\right)} f_{j}^{\left(n_{l}\right)}(t)-b_{j}^{\left(n_{l}+1\right)}\right), i=1,2, \ldots, n_{\text {out }}
$$

Here a simple 3-layer feed-forward fully-connected neural network is considered and is illustrated in Fig. 1.

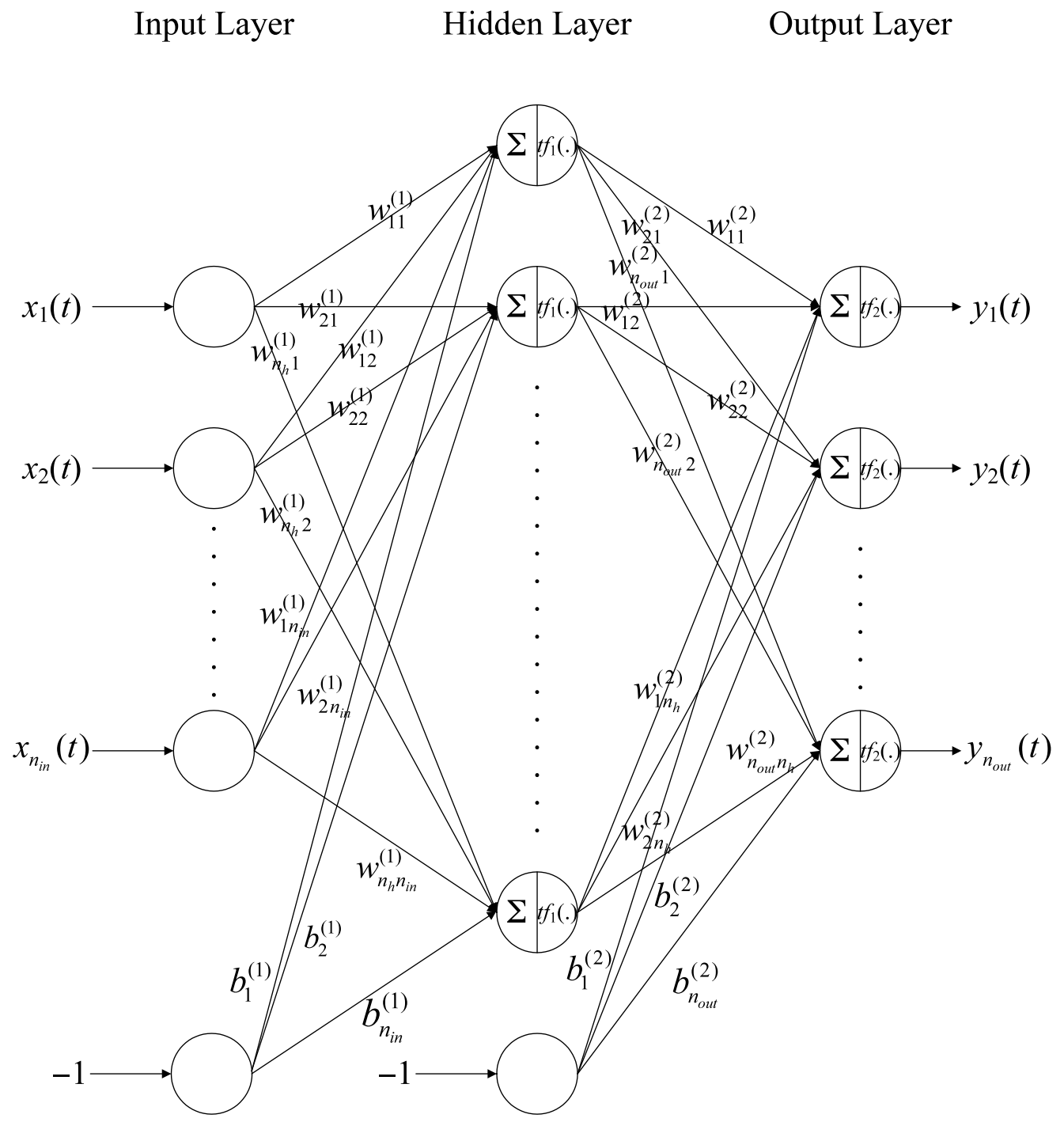

Fig. 1. 3-layer fully-connected feed-forward neural network.

\section{MeChanisms of NN-BASED Classifiers}

In this section, six NN-based classifiers namely one-against-all, weighted one-against-all, binary coded, parallel-structured, weighted parallel-structured and tree-structured, are introduced to classify the feature patterns. In the following, the input pattern is denoted as $\mathbf{x}(t)=\left[\begin{array}{llll}x_{1}(t) & x_{2}(t) & \ldots & x_{n_{\text {in }}}(t)\end{array}\right]$, 
which is considered as the feature vector of an object to be recognized. The purpose of these classifiers is to group the feature patterns into $M$ classes through supervised learning.

\section{A. One-Against-All Classifier}

A one-against-all classifier is shown in Fig. 2, which can be considered as a multiple-input-singleoutput fully-connected feed-forward NN. It receives the feature pattern $\mathbf{x}(t)$ as input and produces a single value $y(t)$ as output. The target output $y^{d}(t)$ is set to be $i$ when the input pattern $\mathbf{x}(t)$ belongs to class $i$. In other words, the one-against-all classifier is trained such that the output $y(t)$ is as close as possible to $y^{d}(t)$ according to the class of the feature pattern $\mathbf{x}(t)$.

During the operation, the feature pattern is classified as of class $j$ which is obtained by

$$
j=\arg \min _{i}\{|y(t)-i| \mid i \in\{1,2, \ldots, M\}\}
$$

where $|\cdot|$ is the absolute value operator. If the set $j$ has more than one element, the first element is considered as the recognized class label.

The one-against-all classifier has a simple structure. However, it is less flexible and retraining is required when additional classes are introduced. Also, when the number of classes increases, the training time increases accordingly. For a large-scale classification problem (for example, with large dataset, large number of classes and/or high dimensional input features), the number of hidden nodes and/or layers have to be increased to achieve an acceptable recognition accuracy.

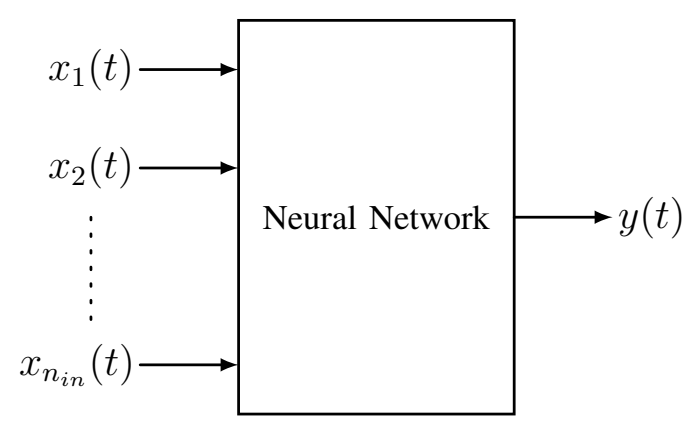

Fig. 2. NN-based one-against-all classifier.

\section{B. Weighted One-Against-All Classifier}

A weighted one-against-all classifier is shown in Fig. 3, which can be considered as a multipleinput-multiple-output fully-connected feed-forward NN. It receives a feature pattern $\mathbf{x}(t)$ as input and 
produces a vector $\mathbf{y}(t)=\left[\begin{array}{llll}y_{1}(t) & y_{2}(t) & \ldots & y_{n_{\text {out }}}(t)\end{array}\right]$ as output where $n_{\text {out }}$ is a non-zero positive integer pre-determined by designers. The target output vector $\mathbf{y}^{d}(t)=\left[\begin{array}{llll}y_{1}^{d}(t) & y_{2}^{d}(t) & \ldots & y_{n_{\text {out }}}^{d}(t)\end{array}\right]$ is set to be $\mathbf{w}_{i}=\left[\begin{array}{llll}w_{i 1} & w_{i 2} & \ldots & w_{i n_{\text {out }}}\end{array}\right], i=1,2, \ldots, M$, which is a predefined constant vector to be determined, when the input pattern $\mathbf{x}(t)$ belongs to class $i$. During the operation, the input pattern is classified as of class $j$ which is obtained by

$$
j=\arg \min _{i}\left\{\left\|\mathbf{y}(t)-\mathbf{w}_{i}\right\| \mid i \in\{1,2, \ldots, M\}\right\}
$$

where $\|\cdot\|$ denotes the $l^{2}$ norm (i.e. Euclidean norm). If the set $j$ has more than one element, the first element is considered as the recognized class label.

Compared with the one-against-all classifier, it offers a relatively higher flexibility to assign the target output, which could improve the recognition accuracy by examining more than one output. As weighted one-against-all classifier is based on the one-against-all classifier, it inherits the same limitations in terms of flexibility, scalability and complexity as in one-against-all Classifier discussed in Section III.B.

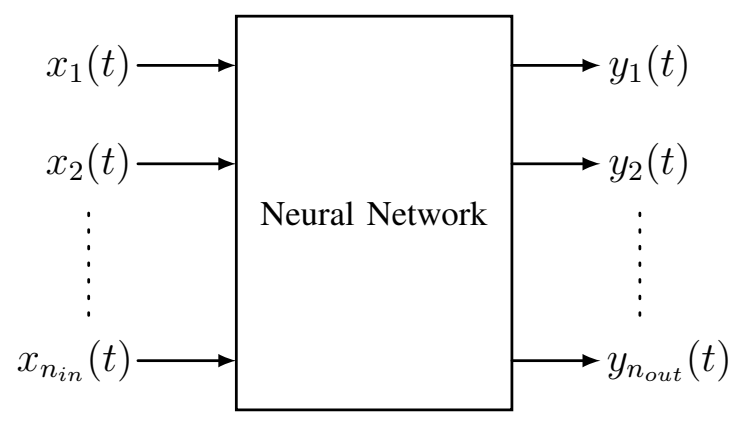

Fig. 3. NN-based weighted one-against-all or binary-coded classifier.

\section{Binary-Coded Classifier}

A binary-coded classifier is shown in Fig. 3, which can be considered as a multiple-input-multipleoutput fully-connected feed-forward NN. It receives a feature pattern $\mathbf{x}(t)$ as input and produces a vector $\mathbf{y}(t)=\left[\begin{array}{llll}y_{1}(t) & y_{2}(t) & \ldots & y_{n_{\text {out }}}(t)\end{array}\right]$ as output where $n_{\text {out }}=\left\lceil\frac{\log M}{\log 2}\right\rceil,\lceil\cdot\rceil$ denotes the ceiling operator rounding up the argument to the nearest integer. To reduce the number of outputs of the NN, binary string is employed to represent the class of the input patterns. Class $i, i=1,2, \ldots, M$, is represented by an $n_{\text {out }}$-bit binary string. For example, assuming that $M=18$, a 5-bit binary string 
is employed to represent the class of input patterns; class 1 is represented by ' 00001 ', class 2 is represented by '00010' and so on. The target output vector $\mathbf{y}^{d}(t)=\left[\begin{array}{llll}y_{1}^{d}(t) & y_{2}^{d}(t) & \ldots & y_{n_{\text {out }}}^{d}(t)\end{array}\right]$ is set to be $\mathbf{w}_{i}=\left[\begin{array}{llll}w_{i 1} & w_{i 2} & \ldots & w_{\text {in out }}\end{array}\right], i=1,2, \ldots, M$, which is the binary representation of $i$.

The binary-coded classifier is a subset of the weighted one-against-all classifier. When the weight $\mathbf{w}_{i}$ of the weighted one-against-all classifier is chosen to be a binary string, the classifier is configured as the binary-coded classifier. During the operation, the input pattern is recognized as of class $j$ based on $(5)$.

\section{Parallel-Structured Classifier}

A parallel-structured classifier is shown in Fig. 4, which consists of $M n_{i n}$-input- $n_{i n}$-output fullyconnected feed-forward NNs. Fig. 4 shows that the purpose of the $i^{\text {th }} \mathrm{NN}$ is to recognize the input patterns of class $i$. To realize this purpose, the training objective is that the output of the NN corresponding to class $i$ is the same as the input patterns of class $i$, i.e., the target output vector $\mathbf{y}^{d}(t)$ is set to be $\mathbf{x}(t)$ such that the characteristic of input patterns of class $i$ can be learnt. Consequently, it is expected that the difference between the input and output vector of the $i^{\text {th }} \mathrm{NN}$ would be very small if the input patterns are of class $i$ but relatively larger if the input pattens are not of class $i$. The class determiner in Fig. 4 will determine the input patten to be of class $i$ if the $i^{\text {th }}$ NN produces the least input-output difference. During the operation, the feature pattern is classified as class $j$ which is obtained by

$$
j=\arg \min _{i}\left\{\left\|\mathbf{y}_{i}(t)-\mathbf{x}(t)\right\| \mid i \in\{1,2, \ldots, M\}\right\}
$$

If the set $j$ has more than one element, the first element is considered as the recognized class label.

The $i^{t h} \mathrm{NN}$ is trained with the feature patterns of class $i$ implying that the complexity of the NN is lower compared with those in one-against-all, weighted one-against-all and binary-coded classifier that feature patterns of all classes are used for training the NN. Moreover, it is more flexible to add extra classes and retraining of all existing NNs is not necessary. It is thus more suitable to handle large-scale recognition problem. 


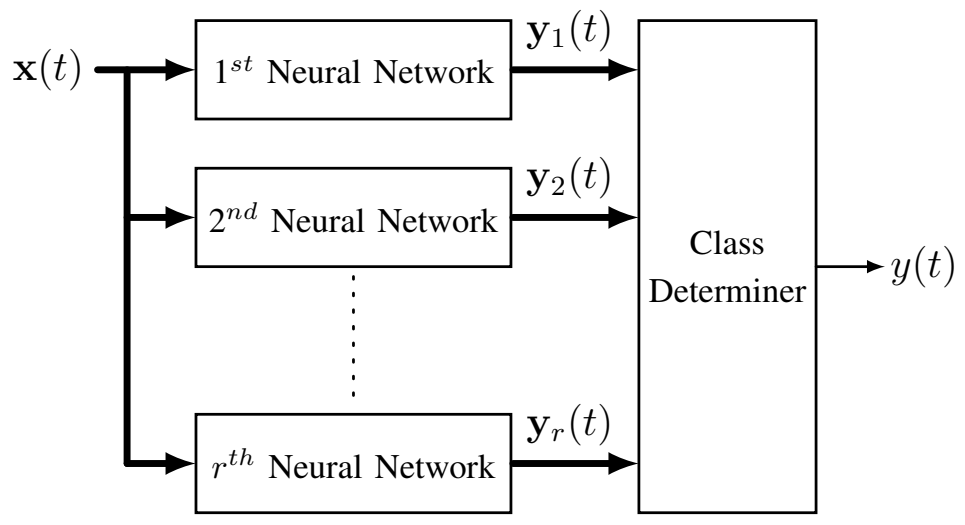

Fig. 4. NN-based parallel-structured classifier.

\section{E. Weighted Parallel-Structured Classifier}

A weighted parallel-structured classifier is a variant of parallel-structured classifier, which consists of $\left\lceil\frac{M}{G}\right\rceil n_{i n}$-input- $n_{i n}$-output fully-connected feed-forward NNs. Each NN in the parallel-structured classifier is able to learn the characteristic of one single class of input patterns and the recognition is realized by looking into the least input-output difference. The weighted parallel-structured classifier allows each $\mathrm{NN}$ to learn the characteristic of more than one class of feature patterns such that each $\mathrm{NN}$ can classify more than one class. It reduces the number of NNs to implement the weighted parallelstructured classifier.

Let $G \leq M$ be the number of classes recognized by each NN. The $i^{t h} \mathrm{NN}$ is trained such that the target output vector $\mathbf{y}^{d}(t)$ is set to be $\mathbf{W}_{k} \mathbf{x}(t)$ where $k=(i-1)\left\lceil\frac{M}{G}\right\rceil+1,(i-1)\left\lceil\frac{M}{G}\right\rceil+2$, $\ldots,(i-1)\left\lceil\frac{M}{G}\right\rceil+G, i=1,2, \ldots,\left\lceil\frac{M}{G}\right\rceil$, when the feature pattern $\mathbf{x}(t)$ belongs to class $k ; \mathbf{W}_{k}=$ $\operatorname{diag}\left\{w_{k 1}, w_{k 2}, \ldots, w_{k n_{i n}}\right\}$ is a constant matrix determined by the designers. Consequently, when the input pattern $\mathbf{x}(t)$ is input to the $i^{t h} \mathrm{NN}$, the $l^{2}$ norm of the difference between the weighted input and output, i.e., $\left\|\mathbf{y}_{i}(t)-\mathbf{W}_{k} \mathbf{x}(t)\right\|$ should be very small when $\mathbf{x}(t)$ belongs to class $k$, otherwise, a relatively larger $l^{2}$ norm of the difference should be obtained. The class determiner will determine the class of the input pattern based on the least $l^{2}$ norm of the difference.

During the operation, the feature pattern is classified as of class $j$ which is obtained by

$$
\begin{aligned}
j= & \arg \min _{k}\left\{\left\|\mathbf{y}_{i}(t)-\mathbf{W}_{k} \mathbf{x}(t)\right\| \mid i \in\left\{1,2, \ldots,\left\lceil\frac{M}{G}\right\rceil\right\}\right. \\
& \left.k \in\left\{(i-1)\left\lceil\frac{M}{G}\right\rceil+1,(i-1)\left\lceil\frac{M}{G}\right\rceil+2, \ldots,(i-1)\left\lceil\frac{M}{G}\right\rceil+G\right\}\right\} .
\end{aligned}
$$


If the set $j$ has more than one element, the first element is considered as the recognized class label.

\section{F. Tree-Structured Classifier}

A tree-structured classifier is shown in Fig. 5, which consists of a single group classifier and $\left\lceil\frac{M}{G}\right\rceil$ sub-classifiers making a total of $1+\left\lceil\frac{M}{G}\right\rceil$ NNs. We firstly divide the total number of classes into $\left\lceil\frac{M}{G}\right\rceil$ groups such that each group has $G$ sub-classes. The group classifier is an $n_{i n}$-input- $\left\lceil\frac{M}{G}\right\rceil$-output NN. The group classifier indicates which group the input pattern belongs to and then select the corresponding sub-classifier to perform recognition. During the training, the target output $z_{k}^{d}(t)$ for output $z_{k}(t), k=$

$1,2, \ldots,\left\lceil\frac{M}{G}\right\rceil$, is set to be 1 if the input pattern belongs to group $k$, otherwise, 0 . When output $z^{k}(t)$ of the group classifier is closer to 1 , which suggests that the input pattern belongs to group $k$, the $k^{\text {th }}$ sub-classifier is selected to determine which sub-class the input pattern belongs to in this group.

During the operation, the feature pattern is classified as of group $j$ which is obtained by

$$
j=\arg \min _{k}\left\{\left|z_{k}(t)-1\right| \mid k \in\left\{1,2, \ldots,\left\lceil\frac{M}{G}\right\rceil\right\}\right\}
$$

If the set $j$ has more than one element, the first element is considered as the recognized class label.

After the input pattern is recognized as of group $j$, the $j^{\text {th }}$ sub-classifier indicates which sub-class the input pattern belongs to. The sub-classifier is an $n_{i n}$-input- $G$-output NN. The $l^{\text {th }}$ output of subclassifier being 1 is to indicate the input pattern belongs to sub-class $l$ in group $j$ so that the actual class of the input pattern is $(j-1) G+l$. Based on this mechanism, the target output $y_{k}^{d}(t)$ for output $y_{k}(t), k=1,2, \ldots, G$, is set to be 1 if the input pattern belongs to sub-class $k$, otherwise, 0 .

During the operation, the input pattern is classified as of sub-class $l$ which is obtained by

$$
l=\arg \min _{k}\left\{\left|y_{k}(t)-1\right| \mid k \in\{1,2, \ldots, G\}\right\}
$$

If the set $l$ has more than one element, the first element is considered as the recognized class label.

The tree-structured classifier provides flexibility to add extra classes without retraining the subclassifiers, however, the group classifier has to be retrained. Furthermore, the number of levels can be increased to deal with large-scale recognition problems. As the recognition error propagates to the lower levels, the recognition performance of the upper-level classifiers, i.e., the group classifier, plays an important role to the overall recognition performance of the tree-structured classifier. Unlike 


\begin{tabular}{|c|c|c|c|c|c|}
\hline Classifier & \#NNs & \#outputs & Flexibility & Scalability & Complexity \\
\hline 1 & 1 & 1 & Low & Low & High \\
\hline 2 & 1 & $n_{\text {out }}$ & Low & Low & High \\
\hline 3 & 1 & $\left\lceil\frac{\log M}{\log 2}\right\rceil$ & Low & Low & High \\
\hline 4 & $M$ & $n_{\text {out }}$ & High & High & Low \\
\hline 5 & $\left\lceil\frac{M}{G}\right\rceil$ & $n_{\text {out }}$ & High & High & Medium \\
\hline 6 & $1+\left\lceil\frac{M}{G}\right\rceil$ & $\left\lceil\frac{\log M}{\log 2}\right\rceil$ or $\left\lceil\frac{M}{G}\right\rceil$ & Medium & Medium & Medium \\
\hline
\end{tabular}

TABLE I

COMPARISON OF VARIOUS NN-BASED CLASSIFIERS. CLASSIFIER 1: ONE-AGAINST-ALL CLASSIFIER, CLASSIFIER 2: WEIGHTED ONE-AGAINSY-ALL CLASSIFIER, CLASSIFIER 3: BINARY-CODED CLASSIFIER, CLASSIFIER 4: PARALLEL-STRUCTURED CLASSIFIER, CLASSIFIER 5: PARALLEL-STRUCTURED CLASSIFIER, CLASSIFIER 6: TREE-STRUCTURED CLASSIFIER.

other classifiers introduced above, the processing time for recognition is relatively longer as the lowerlevel classifiers cannot start to work until result has been received from the upper levels. As the sub-classifiers only need to deal with sub-classes, the complexity of $\mathrm{NN}$ is relatively lower compared with the classifiers with a single NN.

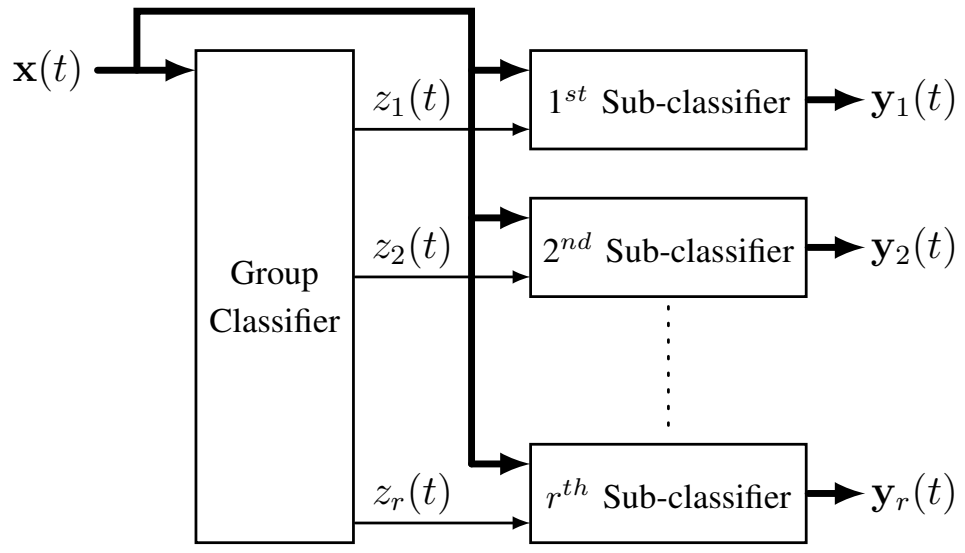

Fig. 5. NN-based tree-structured classifier.

The properties of the NN-classifiers are summarized in Table I, which compares the number of NNs used, number of outputs of NNs, flexibility adding extra classes, scalability in handling large-scale recognition problems and complexity of NNs used in the classifiers.

\section{EXPERIMENTAL RESUlts}

The recognition performance of the introduced $\mathrm{NN}$-based classifiers is investigated using the data collected from a robotic testing platform. The testing platform includes a robot arm Mitsubishi RV- 


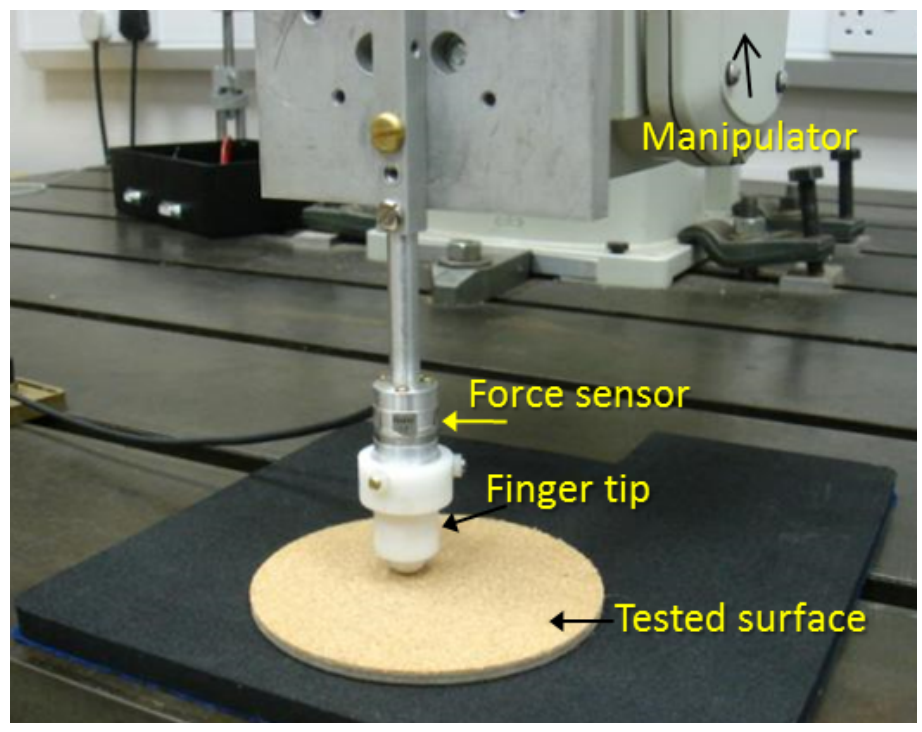

Fig. 6. The test platform.

6SL, a 6-axis force/torque sensor ATI Nano17 (resolution $=0.003 \mathrm{~N}$, sampling rate $=100 \mathrm{~Hz}$ ) and a hemispherical plastic fingertip as shown in Fig 1. During experiments, the fingertip which is rigidly attached to the robot arm was commanded to slide on a selected object surface, keeping the normal force around $2 \mathrm{~N}$. The fingertip was kept perpendicular to the surface all the time. To obtain the dynamic relationship of friction and velocity, within one stroke, the sliding velocity was increased from zero to $15 \mathrm{~mm} / \mathrm{s}$ with a constant acceleration rate of $3 \mathrm{~mm} / \mathrm{s} 2$. In total, surface of 18 materials were investigated and the raw data of fractional force are collected through the force/torque sensor. The 18 materials used in this experiment are summarized in Table II. Each time the fingertip slides along a material surface, 100 numerical values reflecting the material characteristics are collected. 60 sets of data for each material were collected and each set of data contains 100 numerical values. Detailed description of the experiment setup and technical details of raw data collection can be found in [35]. The objective of this experiment is to employ the introduced NN-based classifiers for classifying the 18 materials and comparing their recognition performance.

\section{A. Feature Extraction}

Before applying the introduced NN-based classifiers to recognize the materials, feature vector will be extracted from the raw data consisting of 100 numerical values to reduce the number of data points used for the classification processes. In these experiments, feature vectors of 3,4 and 5 are extracted from the raw data, which will be used as the input of the NN-based classifiers. As a result, the raw data 


\begin{tabular}{|c|l|}
\hline Class label & Material \\
\hline 1 & Un-laminated wood \\
\hline 2 & Fine polished aluminium \\
\hline 3 & Unpolished aluminium \\
\hline 4 & Polished brass \\
\hline 5 & Ceramic plate \\
\hline 6 & Cloth liner \\
\hline 7 & Glass \\
\hline 8 & Artificial leather \\
\hline 9 & Mouse pad (liner surface) \\
\hline 10 & A4 paper \\
\hline 11 & Laminated book cover \\
\hline 12 & Plastic PC mouse \\
\hline 13 & Plastic CD cover \\
\hline 14 & Polymer composite (smooth surface) \\
\hline 15 & Kitchen sponge \\
\hline 16 & Stainless steel knife \\
\hline 17 & Rubber tape \\
\hline 18 & Un-laminated paper package \\
\hline
\end{tabular}

TABLE II

18 MATERIALS USED IN THE EXPERIMENT.

of each pattern (100 numerical values) is represented by 3, 4 or 5 features, which significantly reduces the dimensions of the input features, implying reduced computational demand and implementation complexity.

Details of feature extraction are given below. The raw data of 100 numerical values of each pattern is first divided into $P$ portions where $P=4$ is chosen in this experiment. Denote the raw data of 100 numerical values as $\mathbf{p}=\left[\begin{array}{llll}p_{1} & p_{2} & \ldots & p_{100}\end{array}\right]$, the first to the forth portions of raw data are defined as: $\mathbf{p}_{1}=\left[\begin{array}{llll}p_{1} & p_{2} & \ldots & p_{25}\end{array}\right], \mathbf{p}_{2}=\left[\begin{array}{llll}p_{26} & p_{27} & \ldots & p_{50}\end{array}\right], \mathbf{p}_{3}=\left[\begin{array}{llll}p_{51} & p_{52} & \ldots & p_{75}\end{array}\right]$ and $\mathbf{p}_{4}=\left[\begin{array}{llll}p_{76} & p_{77} & \ldots & p_{100}\end{array}\right]$. Define

$$
\begin{gathered}
f_{1}(\mathbf{z})=\frac{1}{S} \sum_{i=1}^{S} z_{i}, \\
f_{2}(\mathbf{p})=\sum_{i=1}^{4}\left|f_{1}\left(\mathbf{p}_{i+1}\right)-f_{1}\left(\mathbf{p}_{i}\right)\right|,
\end{gathered}
$$




$$
f_{3}(\mathbf{z})=\frac{1}{S-1} \sum_{i=1}^{S}\left(z_{i}-f_{1}(\mathbf{z})\right)^{2}
$$

where $\mathbf{z}=\left[\begin{array}{llll}z_{1} & z_{2} & \ldots & z_{S}\end{array}\right]$.

Feature vectors of 3 to 5 feature points are defined as follows:

Feature vector with 3 points:

$$
\mathbf{x}=\left[\begin{array}{lll}
\sum_{i=1}^{4} f_{1}\left(\mathbf{p}_{i}\right) & 50 f_{2}\left(\mathbf{p}_{i}\right) \quad 50 \sum_{i=1}^{4} f_{3}\left(\mathbf{p}_{i}\right)
\end{array}\right]
$$

Feature vector with 4 points:

$$
\mathbf{x}=\left[\begin{array}{llll}
\sum_{i=1}^{4} f_{1}\left(\mathbf{p}_{i}\right) & 50 f_{2}\left(\mathbf{p}_{i}\right) & 50 \sum_{i=1}^{4} f_{3}\left(\mathbf{p}_{i}\right) & 20 \sum_{i=1}^{4} \sqrt{f_{3}\left(\mathbf{p}_{i}\right)}
\end{array}\right]
$$

Feature vector with 5 points:

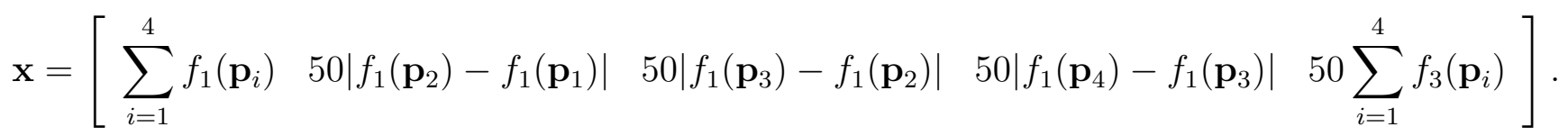

It can be seen from (10) to (12) that $f_{1}(\mathbf{z})$ is the mean of $\mathbf{z}, f_{2}(\mathbf{z})$ is the sum of the difference of the mean of the consecutive portions of raw data, $f_{3}(\mathbf{z})$ is the variance of $\mathbf{z}$.

\section{B. NN-based Classification}

The 6 NN-based classifiers are employed to recognize the 18 materials using the feature vectors of 3, 4, and 5 points. The introduced classifiers were implemented on Matlab.The Levenberg-Marquardt back-propagation is used to develop the classifiers by minimizing the mean square error.

In this experiment, recalling that 60 sets of raw data being collected for each material, 40 of them are be used for the training of NNs and 20 of them are used for testing. Various transfer functions and different number of hidden nodes and hidden layers have been tried in this study. In the following, only the appropriate configurations (number of hidden nodes, transfer functions, etc.) which can achieve acceptable recognition accuracy are reported. The linear transfer function is used in the output layer of all classifiers. For comparison purposes, the traditional kNN classifier and naive Bayes classifier are employed for the classification problem. To investigate how the noise influences the recognition performance of the classifiers, which is inevitable in real world, the test dataset contaminated by 
Gaussian white noise with variance of 0.005 is employed. It should be noted that the simulations for all classifiers tested with noisy test dataset are conducted 10 times for fair comparison, as different solutions can be obtained by the Levenberg-Marquardt back-propagation algorithm with different initial guesses. Statistical information of the tests including the average recognition accuracy for individual class, maximum and minimum recognition accuracy and standard deviation of the 10 tests is reported.

In the following, the recognition performance of the $6 \mathrm{NN}$-based classifiers, kNN classifier and naive Bayes classifier for the classification problem subject to noise-free and noisy datasets is reported.

1) One-Against-All Classifiers: An NN with 3 layers as shown in Fig. 1 is employed to implement the one-against-all classifier. The number of hidden nodes was chosen to be 30 and the transfer function of hidden nodes was chosen to be a logarithmic sigmoid transfer function. The recognition accuracy in percentage for the one-against-all classifier with feature vector of 3 to 5 feature points corresponding to each material is summarized in Table V to Table VII.

Referring to these tables, it can be seen that the average testing recognition accuracy is about $96 \%$ for the one-against-all classifier using feature vector of 3 to 5 feature points. However, looking into the testing recognition accuracy of individual material, the one-against-all classifier using 3 feature points offers $80 \%$ recognition accuracy for material 11 while the one-against-all classifier using 4 or 5 feature points offers $85 \%$ testing recognition accuracy in the worst case. It suggests that the feature vector of 3 feature points may not work well with the one-against-all classifier.

The recognition accuracy for the test data subject to Gaussian white noise is shown in Table XXIX to Table XXXI. It can be seen from the tables that the recognition accuracy of the one-against-all classifiers subject to noisy data has declined to about $92 \%, 83 \%$ and $93 \%$ for 3,4 and 5 feature vectors, respectively. The classifier with 4 feature points performs the worst when the noise exists. Also, it is found that materials 12 and 15 are very sensitive to the noise.

2) Weighted One-Against-All Classifiers: An NN with 3 layers is employed to implement the weighted one-against-all classifier. The elements of the weighting vector $\mathbf{w}_{i}$ were all chosen to be $\lceil i-9.5\rceil, i=1,2, \ldots, 18$. The number of hidden nodes was chosen to be 30 and the transfer function of hidden nodes was chosen to be hyperbolic tangent sigmoid transfer function. The recognition accuracy in percentage for the weighted one-against-all classifier with the feature vector of 3 to 5 feature points corresponding to each material is summarized in Table VIII to Table X for noise-free dataset and Table XXXII to Table XXXIV for noisy dataset. 
Referring to Table VIII to Table X, the average testing recognition accuracy is about $96 \%$ for the weighted one-against-all classifier using the feature vector of 3 or 4 feature points. However, the average testing recognition accuracy is improved to about $98 \%$ for the feature vector of 5 feature points. Looking into the worst individual testing recognition accuracy, the weighted one-against-all classifier using feature vector of 3 feature points offers $80 \%$ for material 13 while the weighted one-against-all classifier using 4 or 5 feature points offers $85 \%$ testing recognition accuracy in the worst case. Similar conclusion that the feature vector of 3 feature points may not work effectively can be drawn.

Referring to Table XXXII to Table XXXIV the performance of weighted one-against-all classifier under noisy data has declined to about $85 \%, 85 \%$ and $95 \%$ respectively. Similar observation is found as in the results from one-against-all classifiers as the same mechanism is used on both one-against-all and weighted one-against-all classifiers.

3) Binary-Coded Classifiers: An NN with 3 layers as shown in Fig. 1 is employed to implement the binary-coded classifier. The number of hidden nodes was chosen to be 30 and the transfer function of hidden nodes was chosen to be logarithmic sigmoid transfer function. The recognition accuracy in percentage for the binary-coded classifier with feature vector of 3 to 5 feature points corresponding to each material is summarized in Table XI to Table XIII for noise-free dataset and Table XXXV to Table XXXVII for noisy dataset.

Referring to Table XI to Table XIII, the average testing recognition accuracy for the binary-coded classifier with feature vector of 3 or 4 feature points is $98 \%$ while with feature vector of 5 feature points is about $99 \%$. The worst individual testing recognition accuracy is $95 \%$ for all binary-coded classifier with feature vector of 3 to 5 feature points. Comparing with the one-against-all or the weighted one-against-all classifier, the recognition performance of binary-coded classifier is less sensitive to the number of feature points.

Referring to Table XXXV to Table XXXVII, the recognition performance of binary-coded classifier under noisy data can be observered. The binary-coded classifier is able to offer a relatively higher performance compared with the one-against-all and weighted one-against-all classifiers. Corresponding to the number of feature points as 3, 4 and 5, the average recognition accuracy can achieve about $97 \%$, $94 \%$ and $99 \%$, respectively. It is again showing that the dataset with 4 feature points produces the worst result. It is observed that materials 12 and 15 are the most difficult classes to be recognized but their recognition accuracy can be significantly improved compared with the previous discussed 
classifiers.

4) Parallel-Structured Classifiers: In the parallel-structured classifier, all NNs are with 3 layers where the number of hidden nodes was chosen to be 10 and the transfer function of hidden nodes was chosen to be logarithmic sigmoid transfer function. Compared with the NNs used in the above classifiers, the number of hidden nodes is significantly reduced, which supports the comment in Table I that the complexity of NN is relatively lower. The recognition accuracy in percentage for the parallelstructured classifier with feature vector of 3 to 5 feature points corresponding to each material is summarized in Table XIV to Table XVI for noise-free dataset and Table XXXVIII to Table XL for noisy dataset.

Referring to Table XIV to Table XVI, the individual training and testing recognition accuracy are all $100 \%$ irregardless of the number of feature points used. Of all classifiers, the parallel-structured classifier offers the best recognition performance. Based on the recognition accuracy, it suggests that 3 features points are sufficient for recognition purposes.

Referring to Table XXXVIII to Table XL the performance of parallel-structured classifier under noisy data can be observed. It can be seen from these 3 tables that the parallel-structured classifier is still able to offer a tolerable performance. Corresponding to the number of feature points as 3,4 and 5 , the average recognition accuracy can achieve about 94\%, 93\% and 96\%, respectively. When the noisy dataset is considered, the recognition performance is not as good as but comparable to that of the binary-coded classifiers. Also, materials 12 and 15 are the most difficult classes to be recognized.

5) Weighted Parallel-Structured Classifiers Classifiers: In the parallel-structured classifier, all NNs are with 3 layers where the number of hidden nodes was chosen to be 15, the transfer function of hidden nodes was chosen to be hyperbolic tangent sigmoid transfer function and $G=3$. The weighting vector $\mathbf{w}_{i}$ were chosen as follows.

Feature vector of 3 feature points:

$$
\begin{gathered}
\mathbf{w}_{i}=\left[\begin{array}{lll}
-1 & -1 & -1
\end{array}\right], i=1,4,7,10,13,16 . \\
\mathbf{w}_{i}=\left[\begin{array}{lll}
1 & 1 & -1
\end{array}\right], i=2,5,8,11,14,17 . \\
\mathbf{w}_{i}=\left[\begin{array}{lll}
1 & 1 & 1
\end{array}\right], i=3,6,9,12,15,18 .
\end{gathered}
$$


Feature vector of 4 feature points:

$$
\begin{gathered}
\mathbf{w}_{i}=\left[\begin{array}{llll}
-1 & -1 & -1 & -1
\end{array}\right], i=1,4,7,10,13,16 . \\
\mathbf{w}_{i}=\left[\begin{array}{llll}
1 & 1 & -1 & -1
\end{array}\right], i=2,5,8,11,14,17 . \\
\mathbf{w}_{i}=\left[\begin{array}{llll}
1 & 1 & 1 & 1
\end{array}\right], i=3,6,9,12,15,18 .
\end{gathered}
$$

Feature vector of 5 feature points:

$$
\begin{gathered}
\mathbf{w}_{i}=\left[\begin{array}{ccccc}
-1 & -1 & -1 & -1 & -1
\end{array}\right], i=1,4,7,10,13,16 . \\
\mathbf{w}_{i}=\left[\begin{array}{llllll}
1 & 1 & 1 & -1 & -1
\end{array}\right], i=2,5,8,11,14,17 \\
\mathbf{w}_{i}=\left[\begin{array}{lllll}
1 & 1 & 1 & 1 & 1
\end{array}\right], i=3,6,9,12,15,18 .
\end{gathered}
$$

The recognition accuracy in percentage for the weighted parallel-structured classifier with feature vector of 3 to 5 feature points corresponding to each material is summarized in Table XVII to Table XIX for noise-free dataset and Table XLI to Table XLIII for noisy dataset.

Referring to Table XVII to Table XIX, it can be seen that the weighted parallel-structured classifier with feature vector of 5 feature points offers the best average testing recognition accuracy of about $99 \%$ with the worst individual recognition accuracy of $95 \%$. Although the weighted parallel-structured classifier with feature vector of 3 or 4 feature points does not have a bad performance with an average testing recognition accuracy of about $98 \%$, the individual testing recognition accuracy is $90 \%$ for 3 feature points and $80 \%$ for 4 feature points.

Referring to Table XLI to Table XLIII, the performance of weighted parallel-structured classifier under noisy data can be observed. The weighted parallel-structured classifier is able to offer tolerable average recognition accuracy of about 90\%, 93\% and 97\%, corresponding to 3, 4 and 5 points of feature vectors, respectively.

6) Tree-Structured Classifiers: In the tree-structured classifier, all NNs are with 3 layers where the number of hidden nodes was chosen to be 20 for the group classifier, 5 for each sub-classifier, the transfer function of hidden nodes was chosen to be logarithmic sigmoid transfer function for both the group classifier and sub-classifiers. The number of sub-classes is chosen to be $G=3$. Compared with 
the NNs used in the above classifiers, the number of hidden nodes in the sub-classifier is small as only 3 sub-classes need to be handled. The recognition accuracy in percentage for the parallel-structured classifier with feature vector of 3 to 5 feature points corresponding to each material is summarized in Table XX to Table XXII for noise-free dataset and Table XLIV to Table XLVI for noisy dataset.

Referring to Table XX to Table XXII, the tree-structured classifier with feature vector of 5 feature points offers $100 \%$ training and testing recognition accuracy while the one with 3 or 4 feature points offers about $99 \%$ testing recognition accuracy and the worst individual testing recognition accuracy of $90 \%$.

It can be seen from Table XLIV to Table XLVI that the tree-structured classifier under noisy data is still able to offer a relatively high recognition accuracy. Corresponding to 3,4 and 5 points of feature vectors, the average recognition accuracy of about 98\%, 97\% and 100\%, respectively, can be achieved. Among all NN-based classifiers, the tree-structured classifiers are more robust to the noisy input.

\section{Traditional Classifiers}

In order to show the superiority and adaptability of the NN-based classifiers, two traditional classifiers, namely kNN classifier and the Naive Bayes classifier, are employed to accomplish the classification of the 18 materials using the features vectors of 3,4 , and 5 points.

1) K-Nearest Neighbor Classifier: In this experiment, fixing the k-nearest to 1, the recognition accuracy in percentage for the $\mathrm{kNN}$ classifiers with feature vectors of 3 to 5 feature points are summarized in Table XXIII to Table XXV.

From Table XXIII to Table XXV, it can be seen that the average recognition accuracy for the training dataset is $100 \%$ for 3, 4 and 5 points of feature vectors. However, when test dataset is considered, the kNN classifiers with 3 feature points can achieve average recognition accuracy of about $96 \%$, which is higher than that of the $\mathrm{kNN}$ classifiers with 4 and 5 feature points, which can achieve only $94 \%$ and $90 \%$ of average recognition accuracy.

From Table XLVII to Table XLIX, it can be found that the recognition performance of the kNN classifiers with noisy dataset has declined to some extent. The best average recognition accuracy of about $94 \%$ is obtained for the $\mathrm{kNN}$ classifier the feature vector of 3 points while the average recognition accuracy is dropped to about $88 \%$ and $89 \%$ for the $\mathrm{kNN}$ classifiers with the feature vector of 4 and 5 points, respectively. It is interestingly observed that materials 12 and 15 can be recognized well. 
However, material 17 becomes the most difficult class to be recognized.

2) Naive Bayes Classifier: The recognition accuracy in percentage for the naive Bayes classifier with feature vector of 3 to 5 points is summarized in Table XXVI and Table XXVIII for noise-free dataset. From these 3 tables, it can be seen that the recognition accuracy of the naive Bayes classifier for training dataset can be achieved as $100 \%$ for 3, 4 and 5 points of feature vectors, the recognition accuracy for the data set are, $99 \%, 100 \%$ and $100 \%$.

When noise is considered in the test dataset, the recognition performance is given in Table $\mathrm{L}$ and Table LII. The best recognition accuracy for the best is about $93.9 \%$ which is achieved by the classifier with the feature vector of 5 points. The worst is about $93 \%$ which is achieved by the classifier with the feature vector of 4 points.

\section{Discussion}

Giving an overall picture of the recognition performance, Table III summarizes the overall recognition performance of the $6 \mathrm{NN}$-based classifiers and two traditional classifiers and Table IV summarizes the overall recognition performance under noisy test dataset. In these two tables, the average recognition accuracy is the overall recognition accuracy, which is the average recognition accuracy of all classes; the worst recognition accuracy is the worst recognition accuracy in the 18 classes.

It can be seen from Table III that in general the classifiers with 5 feature points perform better in terms of the worst individual training and testing recognition accuracy, and the average training and testing recognition accuracy. When 5 feature points are considered, the parallel-structured, treestructured classifier and naive Bayes classifer are able to offer the training and testing recognition accuracy of $100 \%$. The second best is the binary-coded classifier which is able to offer the training and testing recognition accuracy around 99\%. The worst one is the one-against-all classifier which is only able to offer a testing recognition accuracy around 96\%. When considering the kNN classifier, the overall recognition accuracy for the training dataset is $100 \%$. However, among all classifiers, the $\mathrm{kNN}$ classifier offers the worst recognition accuracy for the test dataset.

Under the noisy test dataset, referring to Table IV, in general, the recognition performance declines for all classifiers. The overall average recognition accuracy drops below $90 \%$ for weighted one-againstall classifier when feature vector of 3 points is employed; for one-against-all classifier, weighted oneagainst-all classifier and kNN classifier when feature vector of 4 points is employed; for kNN classifier 
when feature vector of 5 points is employed. It is observed that majority of classifiers can obtain better recognition accuracy when feature vector of 5 points is employed. By looking into the details, it can be seen that the tree-structured classifier can obtain the best recognition accuracy. In particular, when feature vector of 5 points is employed, the tree-structured classifier is able to achieve overall average recognition accuracy of $99.7778 \%$, outperforming the rest classifiers. It can also been seen that the tree-structured classifier demonstrate consistent recognition performance subject to noisy input with the smallest standard deviation among all classifiers. The second best is the binary-coded classifier which can obtain the overall average recognition accuracy of $98.8611 \%$ but the standard derivation is more or less 5 times higher than that of the tree-structured classifier. The worst one is the kNN classifier which can obtain the overall average recognition accuracy of $88.6389 \%$ with a significant higher standard deviation. It is interestingly found that the parallel-structured classifier is less sensitive to the number of feature points used, which is able to offer more or less the same overall average recognition accuracy regardless of the number of feature points under noise-free and noisy conditions.

From the summary tables, it can be concluded that the binary-coded classifier and tree-structured classifier are more suitable for the application of material recognition when feature vector of 5 points are used.

\section{CONCLuSiON}

This paper has introduced 6 neural-network-based classifiers (namely one-against-all, weighted oneagainst-all, binary coded, parallel structured, weighted parallel structured, tree-structured classifier) and two traditional classifiers (namely k-nearest neighbor classifier and naive Bayes classifier) to deal with a material classification problem where the data was collected from a robot finger installed with tactile sensors. In total 18 materials have been considered in the experiment. The properties of each classifier have been discussed and its mechanism of performing classification has been detailed. To perform the classification, feature vectors of size 3, 4 and 5 are extracted for each material. Supervised learning approach has been adopted to train the neural-network-based classifier, kNN classifier and naive Bayes classifier for the recognition of materials. The performance of each classifiers has been fully investigated and compared with each other in terms of recognition accuracy. In the noisy-free case, the results has shown that the parallel-structured classifier produces the best performance among all 8 classifiers when 3,4 and 5 feature points are used. However, under the noisy case, the tree-structured 


\begin{tabular}{|c|c|c|c|c|c|}
\cline { 2 - 6 } \multicolumn{1}{c|}{} & \multicolumn{5}{c|}{ Recognition Accuracy (\%) } \\
\hline \#feature points & Classifier & Worst (Training) & Average (Training) & Worst (Testing) & Average (Testing) \\
\hline 3 & 1 & 100 & 100 & 80 & 96.9444 \\
3 & 2 & 92.5 & 98.8889 & 80 & 96.3889 \\
3 & 3 & 97.5 & 99.7222 & 90 & 98.6111 \\
3 & 4 & 100 & 100 & 100 & 100 \\
3 & 5 & 95 & 99.3056 & 90 & 98.0556 \\
3 & 6 & 97.5 & 99.8611 & 90 & 99.1667 \\
3 & 7 & 100 & 100 & 80 & 95.8333 \\
3 & 8 & 100 & 100 & 90 & 96.4444 \\
\hline 4 & 1 & 100 & 100 & 85 & 96.3889 \\
4 & 2 & 97.5 & 99.8611 & 85 & 100 \\
4 & 3 & 97.5 & 99.5833 & 90 & 98.3333 \\
4 & 4 & 100 & 100 & 100 & 98.6111 \\
4 & 5 & 87.5 & 99.0278 & 90 & 93.6111 \\
4 & 6 & 100 & 100 & 70 & 100 \\
4 & 7 & 100 & 100 & 100 & 96.1111 \\
4 & 8 & 100 & 100 & 85 & 98.3333 \\
\hline 5 & 1 & 100 & 100 & 95 & 99.7222 \\
5 & 2 & 100 & 100 & 95 & 100 \\
5 & 3 & 97.5 & 99.8611 & 100 & 99.1667 \\
5 & 4 & 100 & 100 & 95 & 100 \\
5 & 5 & 97.5 & 99.8611 & 40 & 100 \\
\hline 5 & 6 & 100 & 100 & 100 & \\
\hline
\end{tabular}

TABLE III

SUMMARY OF RECOGNITION PERFORMANCE OF THE 6 NN-BASED CLASSIFIERS, KNN CLASSIFIER AND NAIVE BAYES CLASSIFIER UNDER NOISE-FREE DATASET. CLASSIFIER 1: ONE-AGAINST-ALL CLASSIFIER, CLASSIFIER 2: WEIGHTED ONE-AGAINSY-ALL CLASSIFIER, CLASSIFIER 3: BINARY-CODED CLASSIFIER, CLASSIFIER 4: PARALLEL-STRUCTURED CLASSIFIER, CLASSIFIER 5: PARALLEL-STRUCTURED CLASSIFIER, CLASSIFIER 6: TREE-STRUCTURED CLASSIFIER. CLASSIFIER 7: K-NEAREST NEIGHBOR CLASSIFIER, 8: NAIVE BAYES CLASSIFIER

classifier has achieved the best performance among all the classifiers when 3, 4 and 5 feature points are used. 


\begin{tabular}{|c|c|c|c|c|c|}
\cline { 2 - 6 } \multicolumn{1}{c|}{} & \multicolumn{5}{|c|}{ Recognition Accuracy $(\%)$} \\
\hline \#feature points & Classifier & Worst & Average & Best & Std \\
\hline 3 & 1 & 88.3333 & 92.9722 & 96.3889 & 2.7212 \\
3 & 2 & 80.0000 & 85.2500 & 89.4444 & 3.4430 \\
3 & 3 & 94.4444 & 96.6944 & 98.8889 & 1.4593 \\
3 & 4 & 90.0000 & 93.9722 & 96.3889 & 2.2056 \\
3 & 5 & 86.9444 & 90.1944 & 92.5000 & 1.9889 \\
3 & 6 & 96.3889 & 97.8611 & 99.1667 & 0.9679 \\
3 & 7 & 88.6111 & 93.8889 & 98.3333 & 3.3120 \\
3 & 8 & 92.5000 & 93.5278 & 94.1667 & 0.5826 \\
\hline 4 & 1 & 78.6111 & 83.0000 & 86.6667 & 2.8315 \\
4 & 2 & 81.6667 & 85.3889 & 88.6111 & 2.3061 \\
4 & 3 & 90.0000 & 93.9444 & 96.3889 & 2.1650 \\
4 & 4 & 91.1111 & 92.7778 & 93.8889 & 1.0273 \\
4 & 5 & 90.2778 & 93.1667 & 95.2778 & 1.9215 \\
4 & 6 & 95.5556 & 97.3611 & 99.1667 & 1.0499 \\
4 & 7 & 83.6111 & 87.5926 & 92.2222 & 4.5695 \\
4 & 8 & 92.2222 & 93.0278 & 94.1667 & 0.6887 \\
\hline 5 & 1 & 87.7778 & 93.0278 & 97.2222 & 3.2587 \\
5 & 2 & 92.2222 & 94.8333 & 97.5000 & 1.9245 \\
5 & 3 & 96.6667 & 98.8611 & 99.7222 & 1.1066 \\
5 & 4 & 94.7222 & 95.8056 & 96.6667 & 0.6879 \\
5 & 5 & 92.5000 & 96.5833 & 98.8889 & 2.1120 \\
5 & 6 & 99.1667 & 99.7778 & 100.0000 & 0.2869 \\
5 & 7 & 83.3333 & 88.6389 & 92.7778 & 3.0588 \\
5 & 8 & 93.3333 & 93.9167 & 94.4444 & 0.4086 \\
\hline
\end{tabular}

TABLE IV

NOISE:SUMMARY OF RECOGNITION PERFORMANCE OF THE 6 NN-BASED CLASSIFIERS, KNN CLASSIFIER AND NAIVE BAYES ClASSIFIER. CLASSIFIER 1: ONE-AGAINST-ALL CLASSIFIER, CLASSIFIER 2: WEIGHTED ONE-AGAINSY-ALL CLASSIFIER, CLASSIFIER 3: BINARY-CODED CLASSIFIER, CLASSIFIER 4: PARALLEL-STRUCTURED CLASSIFIER, CLASSIFIER 5: PARALLEL-STRUCTURED CLASSIFIER, CLASSIFIER 6: TREE-STRUCTURED CLASSIFIER. CLASSIFIER 7: K-NEAREST NEIGHBOR CLASSIFIER, 8: NAIVE BAYES CLASSIFIER 


\section{APPENDIX}

\section{TRAining AND Testing ReCOGNition ACCURACY}

\begin{tabular}{|c|c|c|}
\cline { 2 - 3 } \multicolumn{1}{c|}{} & \multicolumn{2}{c|}{ Recognition Accuracy (\%) } \\
\hline Material & Training & Testing \\
\hline 1 & 100.0000 & 95.0000 \\
\hline 2 & 100.0000 & 90.0000 \\
\hline 3 & 100.0000 & 100.0000 \\
\hline 4 & 100.0000 & 100.0000 \\
\hline 5 & 100.0000 & 100.0000 \\
\hline 6 & 100.0000 & 100.0000 \\
\hline 7 & 100.0000 & 100.0000 \\
\hline 8 & 100.0000 & 95.0000 \\
\hline 9 & 100.0000 & 100.0000 \\
\hline 10 & 100.0000 & 100.0000 \\
\hline 11 & 100.0000 & 80.0000 \\
\hline 12 & 100.0000 & 100.0000 \\
\hline 13 & 100.0000 & 100.0000 \\
\hline 14 & 100.0000 & 95.0000 \\
\hline 15 & 100.0000 & 100.0000 \\
\hline 16 & 100.0000 & 95.0000 \\
\hline 17 & 100.0000 & 95.0000 \\
\hline 18 & 100.0000 & 100.0000 \\
\hline \hline Average & 100.0000 & 96.9444 \\
\hline & & \\
\hline
\end{tabular}

TABLE V

NN-BASED ONE-AGAINST-ALL CLASSIFIER USING 3 FEATURE POINTS. NUMBER OF HIDDEN NODES: 30, TRANSFER FUNCTION OF HIDDEN NODES: LOGARITHMIC SIGMOID TRANSFER FUNCTION. 


\begin{tabular}{|c|c|c|}
\cline { 2 - 3 } \multicolumn{1}{c|}{} & \multicolumn{2}{c|}{ Recognition Accuracy (\%) } \\
\hline Material & Training & Testing \\
\hline 1 & 100.0000 & 100.0000 \\
\hline 2 & 100.0000 & 90.0000 \\
\hline 3 & 100.0000 & 100.0000 \\
\hline 4 & 100.0000 & 85.0000 \\
\hline 5 & 100.0000 & 95.0000 \\
\hline 6 & 100.0000 & 100.0000 \\
\hline 7 & 100.0000 & 100.0000 \\
\hline 8 & 100.0000 & 95.0000 \\
\hline 9 & 100.0000 & 100.0000 \\
\hline 10 & 100.0000 & 90.0000 \\
\hline 11 & 100.0000 & 90.0000 \\
\hline 12 & 100.0000 & 95.0000 \\
\hline 13 & 100.0000 & 95.0000 \\
\hline 14 & 100.0000 & 100.0000 \\
\hline 15 & 100.0000 & 100.0000 \\
\hline 16 & 100.0000 & 100.0000 \\
\hline 17 & 100.0000 & 100.0000 \\
\hline 18 & 100.0000 & 100.0000 \\
\hline \hline Average & 100.0000 & 96.3889 \\
\hline
\end{tabular}

TABLE VI

NN-BASED ONE-AGAINST-ALL CLASSIFIER USING 4 FEATURE POINTS. NUMBER OF HIDDEN NODES: 30, TRANSFER FUNCTION OF HIDDEN NODES: LOGARITHMIC SIGMOID TRANSFER FUNCTION.

\begin{tabular}{|c|c|c|}
\cline { 2 - 3 } \multicolumn{1}{c|}{} & \multicolumn{2}{c|}{ Recognition Accuracy (\%) } \\
\hline Material & Training & Testing \\
\hline 1 & 100.0000 & 90.0000 \\
\hline 2 & 100.0000 & 85.0000 \\
\hline 3 & 100.0000 & 95.0000 \\
\hline 4 & 100.0000 & 95.0000 \\
\hline 5 & 100.0000 & 100.0000 \\
\hline 6 & 100.0000 & 100.0000 \\
\hline 7 & 100.0000 & 100.0000 \\
\hline 8 & 100.0000 & 85.0000 \\
\hline 9 & 100.0000 & 100.0000 \\
\hline 10 & 100.0000 & 100.0000 \\
\hline 11 & 100.0000 & 100.0000 \\
\hline 12 & 100.0000 & 100.0000 \\
\hline 13 & 100.0000 & 95.0000 \\
\hline 14 & 100.0000 & 100.0000 \\
\hline 15 & 100.0000 & 95.0000 \\
\hline 16 & 100.0000 & 100.0000 \\
\hline 17 & 100.0000 & 95.0000 \\
\hline 18 & 100.0000 & 95.0000 \\
\hline \hline Average & 100.0000 & 96.1111 \\
\hline & \multicolumn{1}{|c}{} \\
\hline
\end{tabular}

TABLE VII

NN-BASED ONE-AGAINST-ALL CLASSIFIER USING 5 FEATURE POINTS. NUMBER OF HIDDEN NODES: 30, TRANSFER FUNCTION OF HIDDEN NODES: LOGARITHMIC SIGMOID TRANSFER FUNCTION. 


\begin{tabular}{|c|c|c|}
\cline { 2 - 3 } \multicolumn{1}{c|}{} & \multicolumn{2}{c|}{ Recognition Accuracy (\%) } \\
\hline Material & Training & Testing \\
\hline 1 & 97.5000 & 95.0000 \\
\hline 2 & 92.5000 & 90.0000 \\
\hline 3 & 100.0000 & 95.0000 \\
\hline 4 & 97.5000 & 100.0000 \\
\hline 5 & 100.0000 & 100.0000 \\
\hline 6 & 100.0000 & 100.0000 \\
\hline 7 & 100.0000 & 100.0000 \\
\hline 8 & 100.0000 & 95.0000 \\
\hline 9 & 100.0000 & 100.0000 \\
\hline 10 & 100.0000 & 100.0000 \\
\hline 11 & 100.0000 & 95.0000 \\
\hline 12 & 100.0000 & 100.0000 \\
\hline 13 & 100.0000 & 80.0000 \\
\hline 14 & 100.0000 & 100.0000 \\
\hline 15 & 100.0000 & 100.0000 \\
\hline 16 & 95.0000 & 90.0000 \\
\hline 17 & 100.0000 & 100.0000 \\
\hline 18 & 97.5000 & 95.0000 \\
\hline \hline Average & 98.8889 & 96.3889 \\
\hline
\end{tabular}

TABLE VIII

NN-BASED WEIGHTED ONE-AGAINST-ALL CLASSIFIER USING 3 FEATURE POINTS. NUMBER OF HIDDEN NODES: 20, TRANSFER FUNCTION OF HIDDEN NODES: HYPERBOLIC TANGENT SIGMOID TRANSFER FUNCTION.

\begin{tabular}{|c|c|c|}
\cline { 2 - 3 } \multicolumn{1}{c|}{} & \multicolumn{2}{c|}{ Recognition Accuracy (\%) } \\
\hline Material & Training & Testing \\
\hline 1 & 100.0000 & 100.0000 \\
\hline 2 & 97.5000 & 90.0000 \\
\hline 3 & 100.0000 & 100.0000 \\
\hline 4 & 100.0000 & 100.0000 \\
\hline 5 & 100.0000 & 100.0000 \\
\hline 6 & 100.0000 & 95.0000 \\
\hline 7 & 100.0000 & 95.0000 \\
\hline 8 & 100.0000 & 90.0000 \\
\hline 9 & 100.0000 & 100.0000 \\
\hline 10 & 100.0000 & 100.0000 \\
\hline 11 & 100.0000 & 90.0000 \\
\hline 12 & 100.0000 & 95.0000 \\
\hline 13 & 100.0000 & 100.0000 \\
\hline 14 & 100.0000 & 100.0000 \\
\hline 15 & 100.0000 & 100.0000 \\
\hline 16 & 100.0000 & 95.0000 \\
\hline 17 & 100.0000 & 85.0000 \\
\hline 18 & 100.0000 & 100.0000 \\
\hline \hline Average & 99.8611 & 96.3889 \\
\hline
\end{tabular}

TABLE IX

NN-BASED WEIGHTED ONE-AGAINST-ALL CLASSIFIER USING 4 FEATURE POINTS. NUMBER OF HIDDEN NODES: 20, TRANSFER FUNCTION OF HIDDEN NODES: HYPERBOLIC TANGENT SIGMOID TRANSFER FUNCTION. 


\begin{tabular}{|c|c|c|}
\cline { 2 - 3 } \multicolumn{1}{c|}{} & \multicolumn{2}{l|}{ Recognition Accuracy (\%) } \\
\hline Material & Training & Testing \\
\hline 1 & 100.0000 & 95.0000 \\
\hline 2 & 100.0000 & 100.0000 \\
\hline 3 & 100.0000 & 100.0000 \\
\hline 4 & 100.0000 & 100.0000 \\
\hline 5 & 100.0000 & 100.0000 \\
\hline 6 & 100.0000 & 100.0000 \\
\hline 7 & 100.0000 & 95.0000 \\
\hline 8 & 100.0000 & 95.0000 \\
\hline 9 & 100.0000 & 100.0000 \\
\hline 10 & 100.0000 & 95.0000 \\
\hline 11 & 100.0000 & 100.0000 \\
\hline 12 & 100.0000 & 100.0000 \\
\hline 13 & 100.0000 & 100.0000 \\
\hline 14 & 100.0000 & 100.0000 \\
\hline 15 & 100.0000 & 95.0000 \\
\hline 16 & 100.0000 & 100.0000 \\
\hline 17 & 100.0000 & 95.0000 \\
\hline 18 & 100.0000 & 100.0000 \\
\hline \hline Average & 100.0000 & 98.3333 \\
\hline
\end{tabular}

TABLE X

NN-BASED WEIGHTED ONE-AGAINST-ALL CLASSIFIER USING 5 FEATURE POINTS. NUMBER OF HIDDEN NODES: 20, TRANSFER FUNCTION OF HIDDEN NODES: HYPERBOLIC TANGENT SIGMOID TRANSFER FUNCTION.

\begin{tabular}{|c|c|c|}
\cline { 2 - 3 } \multicolumn{1}{c|}{} & \multicolumn{2}{l|}{ Recognition Accuracy (\%) } \\
\hline Material & Training & Testing \\
\hline 1 & 100.0000 & 100.0000 \\
\hline 2 & 100.0000 & 100.0000 \\
\hline 3 & 100.0000 & 100.0000 \\
\hline 4 & 100.0000 & 100.0000 \\
\hline 5 & 100.0000 & 100.0000 \\
\hline 6 & 100.0000 & 100.0000 \\
\hline 7 & 100.0000 & 100.0000 \\
\hline 8 & 97.5000 & 95.0000 \\
\hline 9 & 100.0000 & 100.0000 \\
\hline 10 & 100.0000 & 100.0000 \\
\hline 11 & 97.5000 & 90.0000 \\
\hline 12 & 100.0000 & 100.0000 \\
\hline 13 & 100.0000 & 95.0000 \\
\hline 14 & 100.0000 & 100.0000 \\
\hline 15 & 100.0000 & 100.0000 \\
\hline 16 & 100.0000 & 100.0000 \\
\hline 17 & 100.0000 & 95.0000 \\
\hline 18 & 100.0000 & 100.0000 \\
\hline \hline Average & 99.7222 & 98.6111 \\
\hline & \multicolumn{2}{|}{} \\
\hline
\end{tabular}

TABLE XI

NN-BASED BINARY-CODED-OUTPUT CLASSIFIER USING 3 FEATURE POINTS. NUMBER OF HIDDEN NODES: 30, TRANSFER FUNCTION OF HIDDEN NODES: LOGARITHMIC SIGMOID TRANSFER FUNCTION. 


\begin{tabular}{|c|c|c|}
\cline { 2 - 3 } \multicolumn{1}{c|}{} & \multicolumn{2}{c|}{ Recognition Accuracy (\%) } \\
\hline Material & Training & Testing \\
\hline 1 & 97.5000 & 100.0000 \\
\hline 2 & 100.0000 & 100.0000 \\
\hline 3 & 97.5000 & 100.0000 \\
\hline 4 & 97.5000 & 100.0000 \\
\hline 5 & 100.0000 & 100.0000 \\
\hline 6 & 100.0000 & 100.0000 \\
\hline 7 & 100.0000 & 100.0000 \\
\hline 8 & 100.0000 & 100.0000 \\
\hline 9 & 100.0000 & 100.0000 \\
\hline 10 & 100.0000 & 100.0000 \\
\hline 11 & 100.0000 & 95.0000 \\
\hline 12 & 100.0000 & 100.0000 \\
\hline 13 & 100.0000 & 90.0000 \\
\hline 14 & 100.0000 & 100.0000 \\
\hline 15 & 100.0000 & 100.0000 \\
\hline 16 & 100.0000 & 100.0000 \\
\hline 17 & 100.0000 & 95.0000 \\
\hline 18 & 100.0000 & 100.0000 \\
\hline \hline Average & 99.5833 & 98.8889 \\
\hline
\end{tabular}

TABLE XII

NN-BASED BINARY-CODED-OUTPUT CLASSIFIER USING 4 FEATURE POINTS. NUMBER OF HIDDEN NODES: 30, TRANSFER FUNCTION OF HIDDEN NODES: LOGARITHMIC SIGMOID TRANSFER FUNCTION.

\begin{tabular}{|c|c|c|}
\cline { 2 - 3 } \multicolumn{1}{c|}{} & \multicolumn{2}{c|}{ Recognition Accuracy (\%) } \\
\hline Material & Training & Testing \\
\hline 1 & 100.0000 & 100.0000 \\
\hline 2 & 100.0000 & 100.0000 \\
\hline 3 & 100.0000 & 100.0000 \\
\hline 4 & 100.0000 & 100.0000 \\
\hline 5 & 100.0000 & 100.0000 \\
\hline 6 & 100.0000 & 100.0000 \\
\hline 7 & 100.0000 & 100.0000 \\
\hline 8 & 100.0000 & 100.0000 \\
\hline 9 & 100.0000 & 100.0000 \\
\hline 10 & 100.0000 & 100.0000 \\
\hline 11 & 100.0000 & 100.0000 \\
\hline 12 & 100.0000 & 100.0000 \\
\hline 13 & 100.0000 & 100.0000 \\
\hline 14 & 100.0000 & 100.0000 \\
\hline 15 & 100.0000 & 100.0000 \\
\hline 16 & 100.0000 & 100.0000 \\
\hline 17 & 100.0000 & 95.0000 \\
\hline 18 & 97.5000 & 100.0000 \\
\hline \hline Average & 99.8611 & 99.7222 \\
\hline
\end{tabular}

TABLE XIII

NN-BASED BINARY-CODED-OUTPUT CLASSIFIER USING 5 FEATURE POINTS. NUMBER OF HIDDEN NODES: 30, TRANSFER FUNCTION OF HIDDEN NODES: LOGARITHMIC SIGMOID TRANSFER FUNCTION. 


\begin{tabular}{|c|c|c|}
\cline { 2 - 3 } \multicolumn{1}{c|}{} & \multicolumn{2}{c|}{ Recognition Accuracy (\%) } \\
\hline Material & Training & Testing \\
\hline 1 & 100.0000 & 100.0000 \\
\hline 2 & 100.0000 & 100.0000 \\
\hline 3 & 100.0000 & 100.0000 \\
\hline 4 & 100.0000 & 100.0000 \\
\hline 5 & 100.0000 & 100.0000 \\
\hline 6 & 100.0000 & 100.0000 \\
\hline 7 & 100.0000 & 100.0000 \\
\hline 8 & 100.0000 & 100.0000 \\
\hline 9 & 100.0000 & 100.0000 \\
\hline 10 & 100.0000 & 100.0000 \\
\hline 11 & 100.0000 & 100.0000 \\
\hline 12 & 100.0000 & 100.0000 \\
\hline 13 & 100.0000 & 100.0000 \\
\hline 14 & 100.0000 & 100.0000 \\
\hline 15 & 100.0000 & 100.0000 \\
\hline 16 & 100.0000 & 100.0000 \\
\hline 17 & 100.0000 & 100.0000 \\
\hline 18 & 100.0000 & 100.0000 \\
\hline \hline Average & 100.0000 & 100.0000 \\
\hline
\end{tabular}

TABLE XIV

NN-BASED PARALLEL-STRUCTURED CLASSIFIER USING 3 FEATURE POINTS. NUMBER OF HIDDEN NODES: 10, TRANSFER FUNCTION OF HIDDEN NODES: LOGARITHMIC SIGMOID TRANSFER FUNCTION.

\begin{tabular}{|c|c|c|}
\cline { 2 - 3 } \multicolumn{1}{c|}{} & \multicolumn{2}{l|}{ Recognition Accuracy (\%) } \\
\hline Material & Training & Testing \\
\hline 1 & 100.0000 & 100.0000 \\
\hline 2 & 100.0000 & 100.0000 \\
\hline 3 & 100.0000 & 100.0000 \\
\hline 4 & 100.0000 & 100.0000 \\
\hline 5 & 100.0000 & 100.0000 \\
\hline 6 & 100.0000 & 100.0000 \\
\hline 7 & 100.0000 & 100.0000 \\
\hline 8 & 100.0000 & 100.0000 \\
\hline 9 & 100.0000 & 100.0000 \\
\hline 10 & 100.0000 & 100.0000 \\
\hline 11 & 100.0000 & 100.0000 \\
\hline 12 & 100.0000 & 100.0000 \\
\hline 13 & 100.0000 & 100.0000 \\
\hline 14 & 100.0000 & 100.0000 \\
\hline 15 & 100.0000 & 100.0000 \\
\hline 16 & 100.0000 & 100.0000 \\
\hline 17 & 100.0000 & 100.0000 \\
\hline 18 & 100.0000 & 100.0000 \\
\hline \hline Average & 100.0000 & 100.0000 \\
\hline & \multicolumn{2}{|}{} \\
\hline
\end{tabular}

TABLE XV

NN-BASED PARALLEL-STRUCTURED CLASSIFIER USING 4 FEATURE POINTS. NUMBER OF HIDDEN NODES: 10, TRANSFER FUNCTION OF HIDDEN NODES: LOGARITHMIC SIGMOID TRANSFER FUNCTION. 


\begin{tabular}{|c|c|c|}
\cline { 2 - 3 } \multicolumn{1}{c|}{} & \multicolumn{2}{c|}{ Recognition Accuracy (\%) } \\
\hline Material & Training & Testing \\
\hline 1 & 100.0000 & 100.0000 \\
\hline 2 & 100.0000 & 100.0000 \\
\hline 3 & 100.0000 & 100.0000 \\
\hline 4 & 100.0000 & 100.0000 \\
\hline 5 & 100.0000 & 100.0000 \\
\hline 6 & 100.0000 & 100.0000 \\
\hline 7 & 100.0000 & 100.0000 \\
\hline 8 & 100.0000 & 100.0000 \\
\hline 9 & 100.0000 & 100.0000 \\
\hline 10 & 100.0000 & 100.0000 \\
\hline 11 & 100.0000 & 100.0000 \\
\hline 12 & 100.0000 & 100.0000 \\
\hline 13 & 100.0000 & 100.0000 \\
\hline 14 & 100.0000 & 100.0000 \\
\hline 15 & 100.0000 & 100.0000 \\
\hline 16 & 100.0000 & 100.0000 \\
\hline 17 & 100.0000 & 100.0000 \\
\hline 18 & 100.0000 & 100.0000 \\
\hline \hline Average & 100.0000 & 100.0000 \\
\hline
\end{tabular}

TABLE XVI

NN-BASED PARALLEL-STRUCTURED CLASSIFIER USING 5 FEATURE POINTS. NUMBER OF HIDDEN NODES: 10, TRANSFER FUNCTION OF HIDDEN NODES: LOGARITHMIC SIGMOID TRANSFER FUNCTION.

\begin{tabular}{|c|c|c|}
\cline { 2 - 3 } \multicolumn{1}{c|}{} & \multicolumn{2}{l|}{ Recognition Accuracy (\%) } \\
\hline Material & Training & Testing \\
\hline 1 & 95.0000 & 95.0000 \\
\hline 2 & 100.0000 & 100.0000 \\
\hline 3 & 100.0000 & 95.0000 \\
\hline 4 & 95.0000 & 95.0000 \\
\hline 5 & 97.5000 & 95.0000 \\
\hline 6 & 100.0000 & 100.0000 \\
\hline 7 & 100.0000 & 100.0000 \\
\hline 8 & 100.0000 & 100.0000 \\
\hline 9 & 100.0000 & 100.0000 \\
\hline 10 & 100.0000 & 100.0000 \\
\hline 11 & 100.0000 & 90.0000 \\
\hline 12 & 100.0000 & 100.0000 \\
\hline 13 & 100.0000 & 100.0000 \\
\hline 14 & 100.0000 & 100.0000 \\
\hline 15 & 100.0000 & 100.0000 \\
\hline 16 & 100.0000 & 100.0000 \\
\hline 17 & 100.0000 & 95.0000 \\
\hline 18 & 100.0000 & 100.0000 \\
\hline \hline Average & 99.3056 & 98.0556 \\
\hline & \multicolumn{2}{|}{} \\
\hline
\end{tabular}

TABLE XVII

NN-BASED WEIGHTED PARALLEL-STRUCTURED CLASSIFIER USING 3 FEATURE POINTS. NUMBER OF HIDDEN NODES: 15, TRANSFER FUNCTION OF HIDDEN NODES: HYPERBOLIC TANGENT SIGMOID TRANSFER FUNCTION. 


\begin{tabular}{|c|c|c|}
\cline { 2 - 3 } \multicolumn{1}{c|}{} & \multicolumn{2}{c|}{ Recognition Accuracy (\%) } \\
\hline Material & Training & Testing \\
\hline 1 & 100.0000 & 100.0000 \\
\hline 2 & 100.0000 & 100.0000 \\
\hline 3 & 100.0000 & 100.0000 \\
\hline 4 & 100.0000 & 100.0000 \\
\hline 5 & 100.0000 & 100.0000 \\
\hline 6 & 100.0000 & 95.0000 \\
\hline 7 & 100.0000 & 100.0000 \\
\hline 8 & 100.0000 & 100.0000 \\
\hline 9 & 100.0000 & 100.0000 \\
\hline 10 & 100.0000 & 100.0000 \\
\hline 11 & 87.5000 & 80.0000 \\
\hline 12 & 100.0000 & 100.0000 \\
\hline 13 & 100.0000 & 100.0000 \\
\hline 14 & 100.0000 & 100.0000 \\
\hline 15 & 100.0000 & 100.0000 \\
\hline 16 & 100.0000 & 100.0000 \\
\hline 17 & 95.0000 & 95.0000 \\
\hline 18 & 100.0000 & 100.0000 \\
\hline \hline Average & 99.0278 & 98.3333 \\
\hline
\end{tabular}

TABLE XVIII

NN-BASED WEIGHTED PARALLEL-STRUCTURED CLASSIFIER USING 4 FEATURE POINTS. NUMBER OF HIDDEN NODES: 15, TRANSFER FUNCTION OF HIDDEN NODES: HYPERBOLIC TANGENT SIGMOID TRANSFER FUNCTION.

\begin{tabular}{|c|c|c|}
\cline { 2 - 3 } \multicolumn{1}{c|}{} & \multicolumn{2}{c|}{ Recognition Accuracy (\%) } \\
\hline Material & Training & Testing \\
\hline 1 & 100.0000 & 95.0000 \\
\hline 2 & 100.0000 & 100.0000 \\
\hline 3 & 100.0000 & 100.0000 \\
\hline 4 & 97.5000 & 95.0000 \\
\hline 5 & 100.0000 & 100.0000 \\
\hline 6 & 100.0000 & 95.0000 \\
\hline 7 & 100.0000 & 100.0000 \\
\hline 8 & 100.0000 & 100.0000 \\
\hline 9 & 100.0000 & 100.0000 \\
\hline 10 & 100.0000 & 100.0000 \\
\hline 11 & 100.0000 & 100.0000 \\
\hline 12 & 100.0000 & 100.0000 \\
\hline 13 & 100.0000 & 100.0000 \\
\hline 14 & 100.0000 & 100.0000 \\
\hline 15 & 100.0000 & 100.0000 \\
\hline 16 & 100.0000 & 100.0000 \\
\hline 17 & 100.0000 & 100.0000 \\
\hline 18 & 100.0000 & 100.0000 \\
\hline \hline Average & 99.8611 & 99.1667 \\
\hline & \multicolumn{1}{|c}{} \\
\hline
\end{tabular}

TABLE XIX

NN-BASED WEIGHTED PARALLEL-STRUCTURED CLASSIFIER USING 5 FEATURE POINTS. NUMBER OF HIDDEN NODES: 15, TRANSFER FUNCTION OF HIDDEN NODES: HYPERBOLIC TANGENT SIGMOID TRANSFER FUNCTION. 


\begin{tabular}{|c|c|c|}
\cline { 2 - 3 } \multicolumn{1}{c|}{} & \multicolumn{2}{c|}{ Recognition Accuracy (\%) } \\
\hline Material & Training & Testing \\
\hline 1 & 100.0000 & 100.0000 \\
\hline 2 & 100.0000 & 100.0000 \\
\hline 3 & 100.0000 & 100.0000 \\
\hline 4 & 100.0000 & 100.0000 \\
\hline 5 & 100.0000 & 100.0000 \\
\hline 6 & 100.0000 & 100.0000 \\
\hline 7 & 100.0000 & 100.0000 \\
\hline 8 & 100.0000 & 100.0000 \\
\hline 9 & 100.0000 & 100.0000 \\
\hline 10 & 100.0000 & 100.0000 \\
\hline 11 & 97.5000 & 90.0000 \\
\hline 12 & 100.0000 & 100.0000 \\
\hline 13 & 100.0000 & 100.0000 \\
\hline 14 & 100.0000 & 100.0000 \\
\hline 15 & 100.0000 & 100.0000 \\
\hline 16 & 100.0000 & 95.0000 \\
\hline 17 & 100.0000 & 100.0000 \\
\hline 18 & 100.0000 & 100.0000 \\
\hline \hline Average & 99.8611 & 99.1667 \\
\hline
\end{tabular}

TABLE XX

NN-BASED TREE-STRUCTURED CLASSIFIER USING 3 FEATURE POINTS. NUMBER OF HIDDEN NODES: 20, TRANSFER FUNCTION OF HIDDEN NODES: HYPERBOLIC TANGENT SIGMOID TRANSFER FUNCTION.

\begin{tabular}{|c|c|c|}
\cline { 2 - 3 } \multicolumn{1}{c|}{} & \multicolumn{2}{c|}{ Recognition Accuracy (\%) } \\
\hline Material & Training & Testing \\
\hline 1 & 100.0000 & 100.0000 \\
\hline 2 & 100.0000 & 100.0000 \\
\hline 3 & 100.0000 & 100.0000 \\
\hline 4 & 100.0000 & 100.0000 \\
\hline 5 & 100.0000 & 100.0000 \\
\hline 6 & 100.0000 & 100.0000 \\
\hline 7 & 100.0000 & 100.0000 \\
\hline 8 & 100.0000 & 100.0000 \\
\hline 9 & 100.0000 & 100.0000 \\
\hline 10 & 100.0000 & 100.0000 \\
\hline 11 & 100.0000 & 90.0000 \\
\hline 12 & 100.0000 & 100.0000 \\
\hline 13 & 100.0000 & 90.0000 \\
\hline 14 & 100.0000 & 100.0000 \\
\hline 15 & 100.0000 & 100.0000 \\
\hline 16 & 100.0000 & 100.0000 \\
\hline 17 & 100.0000 & 95.0000 \\
\hline 18 & 100.0000 & 100.0000 \\
\hline \hline Average & 100.0000 & 98.6111 \\
\hline & \multicolumn{1}{|c|}{} \\
\hline
\end{tabular}

TABLE XXI

NN-BASED TREE-STRUCTURED CLASSIFIER USING 4 FEATURE POINTS. NUMBER OF HIDDEN NODES: 20, TRANSFER FUNCTION OF HIDDEN NODES: HYPERBOLIC TANGENT SIGMOID TRANSFER FUNCTION. 


\begin{tabular}{|c|c|c|}
\cline { 2 - 3 } \multicolumn{1}{c|}{} & \multicolumn{2}{c|}{ Recognition Accuracy (\%) } \\
\hline Material & Training & Testing \\
\hline 1 & 100.0000 & 100.0000 \\
\hline 2 & 100.0000 & 100.0000 \\
\hline 3 & 100.0000 & 100.0000 \\
\hline 4 & 100.0000 & 100.0000 \\
\hline 5 & 100.0000 & 100.0000 \\
\hline 6 & 100.0000 & 100.0000 \\
\hline 7 & 100.0000 & 100.0000 \\
\hline 8 & 100.0000 & 100.0000 \\
\hline 9 & 100.0000 & 100.0000 \\
\hline 10 & 100.0000 & 100.0000 \\
\hline 11 & 100.0000 & 100.0000 \\
\hline 12 & 100.0000 & 100.0000 \\
\hline 13 & 100.0000 & 100.0000 \\
\hline 14 & 100.0000 & 100.0000 \\
\hline 15 & 100.0000 & 100.0000 \\
\hline 16 & 100.0000 & 100.0000 \\
\hline 17 & 100.0000 & 100.0000 \\
\hline 18 & 100.0000 & 100.0000 \\
\hline \hline Average & 100.0000 & 100.0000 \\
\hline
\end{tabular}

TABLE XXII

NN-BASED TREE-STRUCTURED CLASSIFIER USING 5 FEATURE POINTS. NUMBER OF HIDDEN NODES: 20, TRANSFER FUNCTION OF HIDDEN NODES: HYPERBOLIC TANGENT SIGMOID TRANSFER FUNCTION.

\begin{tabular}{|c|c|c|}
\cline { 2 - 3 } \multicolumn{1}{c|}{} & \multicolumn{2}{c|}{ Recognition Accuracy (\%) } \\
\hline Material & Training & Testing \\
\hline 1 & 100.0000 & 95.0000 \\
\hline 2 & 100.0000 & 95.0000 \\
\hline 3 & 100.0000 & 100.0000 \\
\hline 4 & 100.0000 & 95.0000 \\
\hline 5 & 100.0000 & 95.0000 \\
\hline 6 & 100.0000 & 100.0000 \\
\hline 7 & 100.0000 & 100.0000 \\
\hline 8 & 100.0000 & 100.0000 \\
\hline 9 & 100.0000 & 95.0000 \\
\hline 10 & 100.0000 & 100.0000 \\
\hline 11 & 100.0000 & 85.0000 \\
\hline 12 & 100.0000 & 100.0000 \\
\hline 13 & 100.0000 & 100.0000 \\
\hline 14 & 100.0000 & 100.0000 \\
\hline 15 & 100.0000 & 100.0000 \\
\hline 16 & 100.0000 & 95.0000 \\
\hline 17 & 100.0000 & 80.0000 \\
\hline 18 & 100.0000 & 90.0000 \\
\hline \hline Average & 100.0000 & 95.8333 \\
\hline & \multicolumn{1}{|c}{ TABLE } \\
\hline
\end{tabular}

TABLE XXIII

K-NEAREST NEIGHBOR CLASSIFIER USING 3 FEATURE POINTS. 


\begin{tabular}{|c|c|c|}
\cline { 2 - 3 } \multicolumn{1}{c|}{} & \multicolumn{2}{c|}{ Recognition Accuracy (\%) } \\
\hline Material & Training & Testing \\
\hline 1 & 100.0000 & 100.0000 \\
\hline 2 & 100.0000 & 90.0000 \\
\hline 3 & 100.0000 & 100.0000 \\
\hline 4 & 100.0000 & 70.0000 \\
\hline 5 & 100.0000 & 95.0000 \\
\hline 6 & 100.0000 & 100.0000 \\
\hline 7 & 100.0000 & 100.0000 \\
\hline 8 & 100.0000 & 100.0000 \\
\hline 9 & 100.0000 & 100.0000 \\
\hline 10 & 100.0000 & 95.0000 \\
\hline 11 & 100.0000 & 90.0000 \\
\hline 12 & 100.0000 & 100.0000 \\
\hline 13 & 100.0000 & 95.0000 \\
\hline 14 & 100.0000 & 100.0000 \\
\hline 15 & 100.0000 & 100.0000 \\
\hline 16 & 100.0000 & 80.0000 \\
\hline 17 & 100.0000 & 70.0000 \\
\hline 18 & 100.0000 & 100.0000 \\
\hline \hline Average & 100.0000 & 93.6111 \\
\hline
\end{tabular}

TABLE XXIV

K-NEAREST NEIGHBOR CLASSIFIER USING 4 FEATURE POINTS.

\begin{tabular}{|c|c|c|}
\cline { 2 - 3 } \multicolumn{1}{c|}{} & \multicolumn{2}{l|}{ Recognition Accuracy (\%) } \\
\hline Material & Training & Testing \\
\hline 1 & 100.0000 & 100.0000 \\
\hline 2 & 100.0000 & 90.0000 \\
\hline 3 & 100.0000 & 100.0000 \\
\hline 4 & 100.0000 & 95.0000 \\
\hline 5 & 100.0000 & 100.0000 \\
\hline 6 & 100.0000 & 100.0000 \\
\hline 7 & 100.0000 & 100.0000 \\
\hline 8 & 100.0000 & 70.0000 \\
\hline 9 & 100.0000 & 100.0000 \\
\hline 10 & 100.0000 & 100.0000 \\
\hline 11 & 100.0000 & 100.0000 \\
\hline 12 & 100.0000 & 100.0000 \\
\hline 13 & 100.0000 & 80.0000 \\
\hline 14 & 100.0000 & 100.0000 \\
\hline 15 & 100.0000 & 100.0000 \\
\hline 16 & 100.0000 & 70.0000 \\
\hline 17 & 100.0000 & 40.0000 \\
\hline 18 & 100.0000 & 70.0000 \\
\hline \hline Average & 100.0000 & 89.7222 \\
\hline
\end{tabular}

TABLE XXV

K-NEAREST NEIGHBOR CLASSIFIER USING 5 FEATURE POINTS. 


\begin{tabular}{|c|c|c|}
\cline { 2 - 3 } \multicolumn{1}{c|}{} & \multicolumn{2}{c|}{ Recognition Accuracy (\%) } \\
\hline Material & Training & Testing \\
\hline 1 & 100.0000 & 100.0000 \\
\hline 2 & 100.0000 & 100.0000 \\
\hline 3 & 100.0000 & 100.0000 \\
\hline 4 & 100.0000 & 100.0000 \\
\hline 5 & 100.0000 & 100.0000 \\
\hline 6 & 100.0000 & 100.0000 \\
\hline 7 & 100.0000 & 100.0000 \\
\hline 8 & 100.0000 & 100.0000 \\
\hline 9 & 100.0000 & 100.0000 \\
\hline 10 & 100.0000 & 100.0000 \\
\hline 11 & 100.0000 & 90.0000 \\
\hline 12 & 100.0000 & 100.0000 \\
\hline 13 & 100.0000 & 100.0000 \\
\hline 14 & 100.0000 & 100.0000 \\
\hline 15 & 100.0000 & 100.0000 \\
\hline 16 & 100.0000 & 100.0000 \\
\hline 17 & 100.0000 & 100.0000 \\
\hline 18 & 100.0000 & 100.0000 \\
\hline \hline Average & 100.0000 & 99.4444 \\
\hline
\end{tabular}

TABLE XXVI

NAIVE BAYES CLASSIFIER USING 3 FEATURE POINTS.

\begin{tabular}{|c|c|c|}
\cline { 2 - 3 } \multicolumn{1}{c|}{} & \multicolumn{2}{c|}{ Recognition Accuracy (\%) } \\
\hline Material & Training & Testing \\
\hline 1 & 100.0000 & 100.0000 \\
\hline 2 & 100.0000 & 100.0000 \\
\hline 3 & 100.0000 & 100.0000 \\
\hline 4 & 100.0000 & 100.0000 \\
\hline 5 & 100.0000 & 100.0000 \\
\hline 6 & 100.0000 & 100.0000 \\
\hline 7 & 100.0000 & 100.0000 \\
\hline 8 & 100.0000 & 100.0000 \\
\hline 9 & 100.0000 & 100.0000 \\
\hline 10 & 100.0000 & 100.0000 \\
\hline 11 & 100.0000 & 100.0000 \\
\hline 12 & 100.0000 & 100.0000 \\
\hline 13 & 100.0000 & 100.0000 \\
\hline 14 & 100.0000 & 100.0000 \\
\hline 15 & 100.0000 & 100.0000 \\
\hline 16 & 100.0000 & 100.0000 \\
\hline 17 & 100.0000 & 100.0000 \\
\hline 18 & 100.0000 & 100.0000 \\
\hline \hline Average & 100.0000 & 100.0000 \\
\hline
\end{tabular}

TABLE XXVII

NAIVE BAYES Classifier USING 4 FEATURE POINTS. 


\begin{tabular}{|c|c|c|}
\cline { 2 - 3 } \multicolumn{1}{c|}{} & \multicolumn{2}{c|}{ Recognition Accuracy (\%) } \\
\hline Material & Training & Testing \\
\hline 1 & 100.0000 & 100.0000 \\
\hline 2 & 100.0000 & 100.0000 \\
\hline 3 & 100.0000 & 100.0000 \\
\hline 4 & 100.0000 & 100.0000 \\
\hline 5 & 100.0000 & 100.0000 \\
\hline 6 & 100.0000 & 100.0000 \\
\hline 7 & 100.0000 & 100.0000 \\
\hline 8 & 100.0000 & 100.0000 \\
\hline 9 & 100.0000 & 100.0000 \\
\hline 10 & 100.0000 & 100.0000 \\
\hline 11 & 100.0000 & 100.0000 \\
\hline 12 & 100.0000 & 100.0000 \\
\hline 13 & 100.0000 & 100.0000 \\
\hline 14 & 100.0000 & 100.0000 \\
\hline 15 & 100.0000 & 100.0000 \\
\hline 16 & 100.0000 & 100.0000 \\
\hline 17 & 100.0000 & 100.0000 \\
\hline 18 & 100.0000 & 100.0000 \\
\hline \hline Average & 100.0000 & 100.0000 \\
\hline
\end{tabular}

TABLE XXVIII

NAIVE BAYES CLASSIFIER USING 5 FEATURE POINTS.

\begin{tabular}{|c|c|c|c|c|}
\cline { 2 - 5 } \multicolumn{1}{c|}{} & \multicolumn{4}{c|}{ Recognition Accuracy (\%) } \\
\hline Material & Average & Min & Max & Std \\
\hline 1 & 99.5000 & 95.0000 & 100.0000 & 1.5811 \\
\hline 2 & 85.5000 & 80.0000 & 90.0000 & 2.8382 \\
\hline 3 & 90.5000 & 75.0000 & 100.0000 & 7.2457 \\
\hline 4 & 97.0000 & 90.0000 & 100.0000 & 3.4960 \\
\hline 5 & 100.0000 & 100.0000 & 100.0000 & 0.0000 \\
\hline 6 & 100.0000 & 100.0000 & 100.0000 & 0.0000 \\
\hline 7 & 100.0000 & 100.0000 & 100.0000 & 0.0000 \\
\hline 8 & 92.5000 & 90.0000 & 95.0000 & 2.6352 \\
\hline 9 & 99.5000 & 95.0000 & 100.0000 & 1.5811 \\
\hline 10 & 95.5000 & 90.0000 & 100.0000 & 3.6893 \\
\hline 11 & 82.0000 & 75.0000 & 90.0000 & 4.2164 \\
\hline 12 & 59.5000 & 45.0000 & 75.0000 & 9.5598 \\
\hline 13 & 100.0000 & 100.0000 & 100.0000 & 0.0000 \\
\hline 14 & 99.5000 & 95.0000 & 100.0000 & 1.5811 \\
\hline 15 & 80.5000 & 70.0000 & 90.0000 & 7.9757 \\
\hline 16 & 95.0000 & 95.0000 & 95.0000 & 0.0000 \\
\hline 17 & 97.0000 & 95.0000 & 100.0000 & 2.5820 \\
\hline 18 & 100.0000 & 100.0000 & 100.0000 & 0.0000 \\
\hline \hline Average & 92.9722 & 88.3333 & 96.3889 & 2.7212 \\
\hline
\end{tabular}

TABLE XXIX

NN-BASED ONE-AGAINST-ALL CLASSIFIER USING 3 FEATURE POINTS OF NOISY TEST DATASET. NUMBER OF HIDDEN NODES: 30 , TRANSFER FUNCTION OF HIDDEN NODES: LOGARITHMIC SIGMOID TRANSFER FUNCTION. 


\begin{tabular}{|c|c|c|c|c|}
\cline { 2 - 5 } \multicolumn{1}{c|}{} & \multicolumn{4}{c|}{ Recognition Accuracy (\%) } \\
\hline Material & Average & Min & Max & Std \\
\hline 1 & 96.0000 & 90.0000 & 100.0000 & 3.9441 \\
\hline 2 & 87.0000 & 80.0000 & 95.0000 & 6.7495 \\
\hline 3 & 75.5000 & 65.0000 & 80.0000 & 4.9721 \\
\hline 4 & 64.5000 & 50.0000 & 75.0000 & 7.9757 \\
\hline 5 & 96.0000 & 95.0000 & 100.0000 & 2.1082 \\
\hline 6 & 99.0000 & 95.0000 & 100.0000 & 2.1082 \\
\hline 7 & 100.0000 & 100.0000 & 100.0000 & 0.0000 \\
\hline 8 & 95.0000 & 95.0000 & 95.0000 & 0.0000 \\
\hline 9 & 100.0000 & 100.0000 & 100.0000 & 0.0000 \\
\hline 10 & 82.0000 & 75.0000 & 95.0000 & 5.8689 \\
\hline 11 & 86.5000 & 75.0000 & 95.0000 & 5.2967 \\
\hline 12 & 19.0000 & 10.0000 & 25.0000 & 6.1464 \\
\hline 13 & 95.0000 & 95.0000 & 95.0000 & 0.0000 \\
\hline 14 & 99.0000 & 95.0000 & 100.0000 & 2.1082 \\
\hline 15 & 0.5000 & 0.0000 & 5.0000 & 1.5811 \\
\hline 16 & 100.0000 & 100.0000 & 100.0000 & 0.0000 \\
\hline 17 & 99.0000 & 95.0000 & 100.0000 & 2.1082 \\
\hline 18 & 100.0000 & 100.0000 & 100.0000 & 0.0000 \\
\hline \hline Average & 83.0000 & 78.6111 & 86.6667 & 2.8315 \\
\hline
\end{tabular}

TABLE XXX

NN-BASED ONE-AGAINST-ALL CLASSIFIER USING 4 FEATURE POINTS OF NOISY TEST DATASET. NUMBER OF HIDDEN NODES: 30 , TRANSFER FUNCTION OF HIDDEN NODES: LOGARITHMIC SIGMOID TRANSFER FUNCTION.

\begin{tabular}{|c|c|c|c|c|}
\cline { 2 - 5 } \multicolumn{1}{c|}{} & \multicolumn{4}{c|}{ Recognition Accuracy (\%) } \\
\hline Material & Average & Min & Max & Std \\
\hline 1 & 84.5000 & 75.0000 & 90.0000 & 4.9721 \\
\hline 2 & 91.5000 & 85.0000 & 100.0000 & 4.7434 \\
\hline 3 & 86.5000 & 75.0000 & 90.0000 & 5.2967 \\
\hline 4 & 96.5000 & 90.0000 & 100.0000 & 3.3747 \\
\hline 5 & 99.5000 & 95.0000 & 100.0000 & 1.5811 \\
\hline 6 & 100.0000 & 100.0000 & 100.0000 & 0.0000 \\
\hline 7 & 98.5000 & 95.0000 & 100.0000 & 2.4152 \\
\hline 8 & 86.0000 & 80.0000 & 90.0000 & 3.9441 \\
\hline 9 & 95.0000 & 90.0000 & 100.0000 & 4.0825 \\
\hline 10 & 100.0000 & 100.0000 & 100.0000 & 0.0000 \\
\hline 11 & 95.0000 & 85.0000 & 100.0000 & 5.2705 \\
\hline 12 & 99.0000 & 95.0000 & 100.0000 & 2.1082 \\
\hline 13 & 97.5000 & 95.0000 & 100.0000 & 2.6352 \\
\hline 14 & 100.0000 & 100.0000 & 100.0000 & 0.0000 \\
\hline 15 & 56.5000 & 40.0000 & 85.0000 & 12.4833 \\
\hline 16 & 98.5000 & 95.0000 & 100.0000 & 2.4152 \\
\hline 17 & 95.0000 & 95.0000 & 95.0000 & 0.0000 \\
\hline 18 & 95.0000 & 90.0000 & 100.0000 & 3.3333 \\
\hline \hline Average & 93.0278 & 87.7778 & 97.2222 & 3.2587 \\
\hline
\end{tabular}

TABLE XXXI

NN-BASED ONE-AGAINST-ALL CLASSIFIER USING 5 FEATURE POINTS OF NOISY TEST DATASET. NUMBER OF HIDDEN NODES: 30, TRANSFER FUNCTION OF HIDDEN NODES: LOGARITHMIC SIGMOID TRANSFER FUNCTION. 


\begin{tabular}{|c|c|c|c|c|}
\cline { 2 - 5 } \multicolumn{1}{c|}{} & \multicolumn{4}{c|}{ Recognition Accuracy (\%) } \\
\hline Material & Average & Min & Max & Std \\
\hline 1 & 76.5000 & 70.0000 & 85.0000 & 5.2967 \\
\hline 2 & 80.5000 & 70.0000 & 90.0000 & 6.4334 \\
\hline 3 & 82.0000 & 75.0000 & 85.0000 & 3.4960 \\
\hline 4 & 97.0000 & 95.0000 & 100.0000 & 2.5820 \\
\hline 5 & 98.5000 & 95.0000 & 100.0000 & 2.4152 \\
\hline 6 & 94.0000 & 90.0000 & 100.0000 & 3.9441 \\
\hline 7 & 100.0000 & 100.0000 & 100.0000 & 0.0000 \\
\hline 8 & 96.0000 & 90.0000 & 100.0000 & 3.9441 \\
\hline 9 & 67.5000 & 45.0000 & 80.0000 & 11.1181 \\
\hline 10 & 99.5000 & 95.0000 & 100.0000 & 1.5811 \\
\hline 11 & 95.5000 & 90.0000 & 100.0000 & 3.6893 \\
\hline 12 & 89.0000 & 80.0000 & 95.0000 & 5.1640 \\
\hline 13 & 86.0000 & 85.0000 & 90.0000 & 2.1082 \\
\hline 14 & 100.0000 & 100.0000 & 100.0000 & 0.0000 \\
\hline 15 & 0.0000 & 0.0000 & 0.0000 & 0.0000 \\
\hline 16 & 89.5000 & 85.0000 & 90.0000 & 1.5811 \\
\hline 17 & 97.5000 & 95.0000 & 100.0000 & 2.6352 \\
\hline 18 & 85.5000 & 80.0000 & 95.0000 & 5.9861 \\
\hline \hline Average & 85.2500 & 80.0000 & 89.4444 & 3.4430 \\
\hline
\end{tabular}

TABLE XXXII

NN-BASED WEIGHTED ONE-AGAINST-ALL CLASSIFIER USING 3 FEATURE POINTS OF NOISY TEST DATASET. NUMBER OF HIDDEN NODES: 20, TRANSFER FUNCTION OF HIDDEN NODES: HYPERBOLIC TANGENT SIGMOID TRANSFER FUNCTION.

\begin{tabular}{|c|c|c|c|c|}
\cline { 2 - 5 } \multicolumn{1}{c|}{} & \multicolumn{4}{c|}{ Recognition Accuracy (\%) } \\
\hline Material & Average & Min & Max & Std \\
\hline 1 & 78.0000 & 70.0000 & 85.0000 & 4.2164 \\
\hline 2 & 87.5000 & 85.0000 & 90.0000 & 2.6352 \\
\hline 3 & 71.5000 & 60.0000 & 80.0000 & 5.2967 \\
\hline 4 & 100.0000 & 100.0000 & 100.0000 & 0.0000 \\
\hline 5 & 96.5000 & 95.0000 & 100.0000 & 2.4152 \\
\hline 6 & 99.0000 & 95.0000 & 100.0000 & 2.1082 \\
\hline 7 & 99.0000 & 95.0000 & 100.0000 & 2.1082 \\
\hline 8 & 92.5000 & 90.0000 & 95.0000 & 2.6352 \\
\hline 9 & 100.0000 & 100.0000 & 100.0000 & 0.0000 \\
\hline 10 & 100.0000 & 100.0000 & 100.0000 & 0.0000 \\
\hline 11 & 95.5000 & 90.0000 & 100.0000 & 2.8382 \\
\hline 12 & 38.5000 & 20.0000 & 55.0000 & 11.5590 \\
\hline 13 & 100.0000 & 100.0000 & 100.0000 & 0.0000 \\
\hline 14 & 100.0000 & 100.0000 & 100.0000 & 0.0000 \\
\hline 15 & 0.0000 & 0.0000 & 0.0000 & 0.0000 \\
\hline 16 & 95.5000 & 95.0000 & 100.0000 & 1.5811 \\
\hline 17 & 83.5000 & 75.0000 & 90.0000 & 4.1164 \\
\hline 18 & 100.0000 & 100.0000 & 100.0000 & 0.0000 \\
\hline \hline Average & 85.3889 & 81.6667 & 88.6111 & 2.3061 \\
\hline
\end{tabular}

TABLE XXXIII

NN-BASED WEIGHTED ONE-AGAINST-ALL CLASSIFIER USING 4 FEATURE POINTS OF NOISY TEST DATASET. NUMBER OF HIDDEN NODES: 20, TRANSFER FUNCTION OF HIDDEN NODES: HYPERBOLIC TANGENT SIGMOID TRANSFER FUNCTION. 


\begin{tabular}{|c|c|c|c|c|}
\cline { 2 - 5 } \multicolumn{1}{c|}{} & \multicolumn{4}{c|}{ Recognition Accuracy (\%) } \\
\hline Material & Average & Min & Max & Std \\
\hline 1 & 91.5000 & 80.0000 & 100.0000 & 5.7975 \\
\hline 2 & 99.0000 & 95.0000 & 100.0000 & 2.1082 \\
\hline 3 & 96.0000 & 85.0000 & 100.0000 & 5.1640 \\
\hline 4 & 100.0000 & 100.0000 & 100.0000 & 0.0000 \\
\hline 5 & 97.5000 & 95.0000 & 100.0000 & 2.6352 \\
\hline 6 & 100.0000 & 100.0000 & 100.0000 & 0.0000 \\
\hline 7 & 96.5000 & 95.0000 & 100.0000 & 2.4152 \\
\hline 8 & 95.5000 & 95.0000 & 100.0000 & 1.5811 \\
\hline 9 & 100.0000 & 100.0000 & 100.0000 & 0.0000 \\
\hline 10 & 100.0000 & 100.0000 & 100.0000 & 0.0000 \\
\hline 11 & 98.0000 & 95.0000 & 100.0000 & 2.5820 \\
\hline 12 & 100.0000 & 100.0000 & 100.0000 & 0.0000 \\
\hline 13 & 100.0000 & 100.0000 & 100.0000 & 0.0000 \\
\hline 14 & 96.5000 & 95.0000 & 100.0000 & 2.4152 \\
\hline 15 & 43.0000 & 35.0000 & 60.0000 & 7.5277 \\
\hline 16 & 100.0000 & 100.0000 & 100.0000 & 0.0000 \\
\hline 17 & 93.5000 & 90.0000 & 95.0000 & 2.4152 \\
\hline 18 & 100.0000 & 100.0000 & 100.0000 & 0.0000 \\
\hline \hline Average & 94.8333 & 92.2222 & 97.5000 & 1.9245 \\
\hline
\end{tabular}

TABLE XXXIV

NN-BASED WEIGHTED ONE-AGAINST-ALL CLASSIFIER USING 5 FEATURE POINTS OF NOISY TEST DATASET. NUMBER OF HIDDEN NODES: 20, TRANSFER FUNCTION OF HIDDEN NODES: HYPERBOLIC TANGENT SIGMOID TRANSFER FUNCTION.

\begin{tabular}{|c|c|c|c|c|}
\cline { 2 - 5 } \multicolumn{1}{c|}{} & \multicolumn{4}{c|}{ Recognition Accuracy (\%) } \\
\hline Material & Average & Min & Max & Std \\
\hline 1 & 100.0000 & 100.0000 & 100.0000 & 0.0000 \\
\hline 2 & 94.0000 & 90.0000 & 100.0000 & 3.1623 \\
\hline 3 & 90.5000 & 80.0000 & 100.0000 & 5.9861 \\
\hline 4 & 100.0000 & 100.0000 & 100.0000 & 0.0000 \\
\hline 5 & 100.0000 & 100.0000 & 100.0000 & 0.0000 \\
\hline 6 & 100.0000 & 100.0000 & 100.0000 & 0.0000 \\
\hline 7 & 99.5000 & 95.0000 & 100.0000 & 1.5811 \\
\hline 8 & 94.5000 & 90.0000 & 95.0000 & 1.5811 \\
\hline 9 & 100.0000 & 100.0000 & 100.0000 & 0.0000 \\
\hline 10 & 100.0000 & 100.0000 & 100.0000 & 0.0000 \\
\hline 11 & 96.5000 & 95.0000 & 100.0000 & 2.4152 \\
\hline 12 & 100.0000 & 100.0000 & 100.0000 & 0.0000 \\
\hline 13 & 95.0000 & 95.0000 & 95.0000 & 0.0000 \\
\hline 14 & 100.0000 & 100.0000 & 100.0000 & 0.0000 \\
\hline 15 & 74.5000 & 65.0000 & 90.0000 & 6.8516 \\
\hline 16 & 100.0000 & 100.0000 & 100.0000 & 0.0000 \\
\hline 17 & 97.0000 & 95.0000 & 100.0000 & 2.5820 \\
\hline 18 & 99.0000 & 95.0000 & 100.0000 & 2.1082 \\
\hline \hline Average & 96.6944 & 94.4444 & 98.8889 & 1.4593 \\
\hline
\end{tabular}

TABLE XXXV

NN-BASED BINARY-CODED-OUTPUT CLASSIFIER USING 3 FEATURE POINTS OF NOISY TEST DATASET. NUMBER OF HIDDEN NODES: 30, TRANSFER FUNCTION OF HIDDEN NODES: LOGARITHMIC SIGMOID TRANSFER FUNCTION. 


\begin{tabular}{|c|c|c|c|c|}
\cline { 2 - 5 } \multicolumn{1}{c|}{} & \multicolumn{4}{c|}{ Recognition Accuracy (\%) } \\
\hline Material & Average & Min & Max & Std \\
\hline 1 & 92.0000 & 80.0000 & 100.0000 & 5.3748 \\
\hline 2 & 99.5000 & 95.0000 & 100.0000 & 1.5811 \\
\hline 3 & 64.5000 & 50.0000 & 75.0000 & 7.9757 \\
\hline 4 & 100.0000 & 100.0000 & 100.0000 & 0.0000 \\
\hline 5 & 100.0000 & 100.0000 & 100.0000 & 0.0000 \\
\hline 6 & 100.0000 & 100.0000 & 100.0000 & 0.0000 \\
\hline 7 & 100.0000 & 100.0000 & 100.0000 & 0.0000 \\
\hline 8 & 100.0000 & 100.0000 & 100.0000 & 0.0000 \\
\hline 9 & 100.0000 & 100.0000 & 100.0000 & 0.0000 \\
\hline 10 & 100.0000 & 100.0000 & 100.0000 & 0.0000 \\
\hline 11 & 98.0000 & 90.0000 & 100.0000 & 3.4960 \\
\hline 12 & 75.0000 & 60.0000 & 90.0000 & 11.0554 \\
\hline 13 & 95.0000 & 95.0000 & 95.0000 & 0.0000 \\
\hline 14 & 100.0000 & 100.0000 & 100.0000 & 0.0000 \\
\hline 15 & 72.5000 & 60.0000 & 80.0000 & 7.9057 \\
\hline 16 & 100.0000 & 100.0000 & 100.0000 & 0.0000 \\
\hline 17 & 94.5000 & 90.0000 & 95.0000 & 1.5811 \\
\hline 18 & 100.0000 & 100.0000 & 100.0000 & 0.0000 \\
\hline \hline Average & 93.9444 & 90.0000 & 96.3889 & 2.1650 \\
\hline
\end{tabular}

TABLE XXXVI

NN-BASED BINARY-CODED-OUTPUT CLASSIFIER USING 4 FEATURE POINTS OF NOISY TEST DATASET. NUMBER OF HIDDEN NODES: 30, TRANSFER FUNCTION OF HIDDEN NODES: LOGARITHMIC SIGMOID TRANSFER FUNCTION.

\begin{tabular}{|c|c|c|c|c|}
\cline { 2 - 5 } \multicolumn{1}{c|}{} & \multicolumn{4}{c|}{ Recognition Accuracy (\%) } \\
\hline Material & Average & Min & Max & Std \\
\hline 1 & 97.5000 & 95.0000 & 100.0000 & 2.6352 \\
\hline 2 & 100.0000 & 100.0000 & 100.0000 & 0.0000 \\
\hline 3 & 99.5000 & 95.0000 & 100.0000 & 1.5811 \\
\hline 4 & 100.0000 & 100.0000 & 100.0000 & 0.0000 \\
\hline 5 & 100.0000 & 100.0000 & 100.0000 & 0.0000 \\
\hline 6 & 100.0000 & 100.0000 & 100.0000 & 0.0000 \\
\hline 7 & 100.0000 & 100.0000 & 100.0000 & 0.0000 \\
\hline 8 & 99.5000 & 95.0000 & 100.0000 & 1.5811 \\
\hline 9 & 100.0000 & 100.0000 & 100.0000 & 0.0000 \\
\hline 10 & 100.0000 & 100.0000 & 100.0000 & 0.0000 \\
\hline 11 & 96.5000 & 90.0000 & 100.0000 & 3.3747 \\
\hline 12 & 100.0000 & 100.0000 & 100.0000 & 0.0000 \\
\hline 13 & 100.0000 & 100.0000 & 100.0000 & 0.0000 \\
\hline 14 & 100.0000 & 100.0000 & 100.0000 & 0.0000 \\
\hline 15 & 94.0000 & 80.0000 & 100.0000 & 6.5828 \\
\hline 16 & 98.0000 & 95.0000 & 100.0000 & 2.5820 \\
\hline 17 & 95.0000 & 95.0000 & 95.0000 & 0.0000 \\
\hline 18 & 99.5000 & 95.0000 & 100.0000 & 1.5811 \\
\hline \hline Average & 98.8611 & 96.6667 & 99.7222 & 1.1066 \\
\hline
\end{tabular}

TABLE XXXVII

NN-BASED BINARY-CODED-OUTPUT CLASSIFIER USING 5 FEATURE POINTS OF NOISY TEST DATASET. NUMBER OF HIDDEN NODES: 30, TRANSFER FUNCTION OF HIDDEN NODES: LOGARITHMIC SIGMOID TRANSFER FUNCTION. 


\begin{tabular}{|c|c|c|c|c|}
\cline { 2 - 5 } \multicolumn{1}{c|}{} & \multicolumn{4}{c|}{ Recognition Accuracy (\%) } \\
\hline Material & Average & Min & Max & Std \\
\hline 1 & 99.5000 & 95.0000 & 100.0000 & 1.5811 \\
\hline 2 & 97.5000 & 95.0000 & 100.0000 & 2.6352 \\
\hline 3 & 72.0000 & 60.0000 & 85.0000 & 6.7495 \\
\hline 4 & 100.0000 & 100.0000 & 100.0000 & 0.0000 \\
\hline 5 & 100.0000 & 100.0000 & 100.0000 & 0.0000 \\
\hline 6 & 100.0000 & 100.0000 & 100.0000 & 0.0000 \\
\hline 7 & 100.0000 & 100.0000 & 100.0000 & 0.0000 \\
\hline 8 & 100.0000 & 100.0000 & 100.0000 & 0.0000 \\
\hline 9 & 97.0000 & 95.0000 & 100.0000 & 2.5820 \\
\hline 10 & 100.0000 & 100.0000 & 100.0000 & 0.0000 \\
\hline 11 & 99.0000 & 95.0000 & 100.0000 & 2.1082 \\
\hline 12 & 75.0000 & 55.0000 & 90.0000 & 11.7851 \\
\hline 13 & 100.0000 & 100.0000 & 100.0000 & 0.0000 \\
\hline 14 & 100.0000 & 100.0000 & 100.0000 & 0.0000 \\
\hline 15 & 51.5000 & 25.0000 & 60.0000 & 12.2588 \\
\hline 16 & 100.0000 & 100.0000 & 100.0000 & 0.0000 \\
\hline 17 & 100.0000 & 100.0000 & 100.0000 & 0.0000 \\
\hline 18 & 100.0000 & 100.0000 & 100.0000 & 0.0000 \\
\hline \hline Average & 93.9722 & 90.0000 & 96.3889 & 2.2056 \\
\hline
\end{tabular}

TABLE XXXVIII

NN-BASED PARALLEL-STRUCTURED CLASSIFIER USING 3 FEATURE POINTS OF NOISY TEST DATASET. NUMBER OF HIDDEN NODES: 10, TRANSFER FUNCTION OF HIDDEN NODES: LOGARITHMIC SIGMOID TRANSFER FUNCTION.

\begin{tabular}{|c|c|c|c|c|}
\cline { 2 - 5 } \multicolumn{1}{c|}{} & \multicolumn{4}{c|}{ Recognition Accuracy (\%) } \\
\hline Material & Average & Min & Max & Std \\
\hline 1 & 95.5000 & 85.0000 & 100.0000 & 4.3780 \\
\hline 2 & 97.5000 & 95.0000 & 100.0000 & 2.6352 \\
\hline 3 & 80.5000 & 75.0000 & 85.0000 & 4.3780 \\
\hline 4 & 100.0000 & 100.0000 & 100.0000 & 0.0000 \\
\hline 5 & 100.0000 & 100.0000 & 100.0000 & 0.0000 \\
\hline 6 & 100.0000 & 100.0000 & 100.0000 & 0.0000 \\
\hline 7 & 100.0000 & 100.0000 & 100.0000 & 0.0000 \\
\hline 8 & 100.0000 & 100.0000 & 100.0000 & 0.0000 \\
\hline 9 & 100.0000 & 100.0000 & 100.0000 & 0.0000 \\
\hline 10 & 100.0000 & 100.0000 & 100.0000 & 0.0000 \\
\hline 11 & 100.0000 & 100.0000 & 100.0000 & 0.0000 \\
\hline 12 & 99.0000 & 95.0000 & 100.0000 & 2.1082 \\
\hline 13 & 100.0000 & 100.0000 & 100.0000 & 0.0000 \\
\hline 14 & 100.0000 & 100.0000 & 100.0000 & 0.0000 \\
\hline 15 & 2.5000 & 0.0000 & 5.0000 & 2.6352 \\
\hline 16 & 100.0000 & 100.0000 & 100.0000 & 0.0000 \\
\hline 17 & 100.0000 & 100.0000 & 100.0000 & 0.0000 \\
\hline 18 & 95.0000 & 90.0000 & 100.0000 & 2.3570 \\
\hline \hline Average & 92.7778 & 91.1111 & 93.8889 & 1.0273 \\
\hline
\end{tabular}

TABLE XXXIX

NN-BASED PARALLEL-STRUCTURED CLASSIFIER USING 4 FEATURE POINTS OF NOISY TEST DATASET. NUMBER OF HIDDEN NODES: 10, TRANSFER FUNCTION OF HIDDEN NODES: LOGARITHMIC SIGMOID TRANSFER FUNCTION. 


\begin{tabular}{|c|c|c|c|c|}
\cline { 2 - 5 } \multicolumn{1}{c|}{} & \multicolumn{4}{c|}{ Recognition Accuracy (\%) } \\
\hline Material & Average & Min & Max & Std \\
\hline 1 & 100.0000 & 100.0000 & 100.0000 & 0.0000 \\
\hline 2 & 100.0000 & 100.0000 & 100.0000 & 0.0000 \\
\hline 3 & 100.0000 & 100.0000 & 100.0000 & 0.0000 \\
\hline 4 & 100.0000 & 100.0000 & 100.0000 & 0.0000 \\
\hline 5 & 100.0000 & 100.0000 & 100.0000 & 0.0000 \\
\hline 6 & 100.0000 & 100.0000 & 100.0000 & 0.0000 \\
\hline 7 & 100.0000 & 100.0000 & 100.0000 & 0.0000 \\
\hline 8 & 100.0000 & 100.0000 & 100.0000 & 0.0000 \\
\hline 9 & 100.0000 & 100.0000 & 100.0000 & 0.0000 \\
\hline 10 & 100.0000 & 100.0000 & 100.0000 & 0.0000 \\
\hline 11 & 99.5000 & 95.0000 & 100.0000 & 1.5811 \\
\hline 12 & 100.0000 & 100.0000 & 100.0000 & 0.0000 \\
\hline 13 & 100.0000 & 100.0000 & 100.0000 & 0.0000 \\
\hline 14 & 100.0000 & 100.0000 & 100.0000 & 0.0000 \\
\hline 15 & 25.0000 & 10.0000 & 40.0000 & 10.8012 \\
\hline 16 & 100.0000 & 100.0000 & 100.0000 & 0.0000 \\
\hline 17 & 100.0000 & 100.0000 & 100.0000 & 0.0000 \\
\hline 18 & 100.0000 & 100.0000 & 100.0000 & 0.0000 \\
\hline \hline Average & 95.8056 & 94.7222 & 96.6667 & 0.6879 \\
\hline
\end{tabular}

TABLE XL

NN-BASED PARALLEL-STRUCTURED CLASSIFIER USING 5 FEATURE POINTS OF NOISY TEST DATASET. NUMBER OF HIDDEN NODES: 10, TRANSFER FUNCTION OF HIDDEN NODES: LOGARITHMIC SIGMOID TRANSFER FUNCTION.

\begin{tabular}{|c|c|c|c|c|}
\cline { 2 - 5 } \multicolumn{1}{c|}{} & \multicolumn{4}{c|}{ Recognition Accuracy (\%) } \\
\hline Material & Average & Min & Max & Std \\
\hline 1 & 67.5000 & 55.0000 & 75.0000 & 6.7700 \\
\hline 2 & 99.0000 & 95.0000 & 100.0000 & 2.1082 \\
\hline 3 & 75.0000 & 70.0000 & 80.0000 & 3.3333 \\
\hline 4 & 91.0000 & 80.0000 & 100.0000 & 5.6765 \\
\hline 5 & 95.0000 & 90.0000 & 100.0000 & 2.3570 \\
\hline 6 & 99.0000 & 95.0000 & 100.0000 & 2.1082 \\
\hline 7 & 100.0000 & 100.0000 & 100.0000 & 0.0000 \\
\hline 8 & 100.0000 & 100.0000 & 100.0000 & 0.0000 \\
\hline 9 & 100.0000 & 100.0000 & 100.0000 & 0.0000 \\
\hline 10 & 100.0000 & 100.0000 & 100.0000 & 0.0000 \\
\hline 11 & 94.5000 & 90.0000 & 100.0000 & 4.3780 \\
\hline 12 & 100.0000 & 100.0000 & 100.0000 & 0.0000 \\
\hline 13 & 100.0000 & 100.0000 & 100.0000 & 0.0000 \\
\hline 14 & 100.0000 & 100.0000 & 100.0000 & 0.0000 \\
\hline 15 & 5.5000 & 0.0000 & 10.0000 & 4.3780 \\
\hline 16 & 99.0000 & 95.0000 & 100.0000 & 2.1082 \\
\hline 17 & 98.0000 & 95.0000 & 100.0000 & 2.5820 \\
\hline 18 & 100.0000 & 100.0000 & 100.0000 & 0.0000 \\
\hline \hline Average & 90.1944 & 86.9444 & 92.5000 & 1.9889 \\
\hline
\end{tabular}

TABLE XLI

NN-BASED WEIGHTED PARALLEL-STRUCTURED CLASSIFIER USING 3 FEATURE POINTS OF NOISY TEST DATASET. NUMBER OF HIDDEN NODES: 15, TRANSFER FUNCTION OF HIDDEN NODES: HYPERBOLIC TANGENT SIGMOID TRANSFER FUNCTION. 


\begin{tabular}{|c|c|c|c|c|}
\cline { 2 - 5 } \multicolumn{1}{c|}{} & \multicolumn{4}{c|}{ Recognition Accuracy (\%) } \\
\hline Material & Average & Min & Max & Std \\
\hline 1 & 99.5000 & 95.0000 & 100.0000 & 1.5811 \\
\hline 2 & 97.5000 & 95.0000 & 100.0000 & 2.6352 \\
\hline 3 & 96.5000 & 95.0000 & 100.0000 & 2.4152 \\
\hline 4 & 99.0000 & 95.0000 & 100.0000 & 2.1082 \\
\hline 5 & 96.5000 & 90.0000 & 100.0000 & 4.1164 \\
\hline 6 & 99.0000 & 95.0000 & 100.0000 & 2.1082 \\
\hline 7 & 100.0000 & 100.0000 & 100.0000 & 0.0000 \\
\hline 8 & 100.0000 & 100.0000 & 100.0000 & 0.0000 \\
\hline 9 & 100.0000 & 100.0000 & 100.0000 & 0.0000 \\
\hline 10 & 99.5000 & 95.0000 & 100.0000 & 1.5811 \\
\hline 11 & 86.5000 & 80.0000 & 95.0000 & 6.2583 \\
\hline 12 & 10.5000 & 0.0000 & 25.0000 & 7.6194 \\
\hline 13 & 100.0000 & 100.0000 & 100.0000 & 0.0000 \\
\hline 14 & 100.0000 & 100.0000 & 100.0000 & 0.0000 \\
\hline 15 & 100.0000 & 100.0000 & 100.0000 & 0.0000 \\
\hline 16 & 99.5000 & 95.0000 & 100.0000 & 1.5811 \\
\hline 17 & 93.0000 & 90.0000 & 95.0000 & 2.5820 \\
\hline 18 & 100.0000 & 100.0000 & 100.0000 & 0.0000 \\
\hline \hline Average & 93.1667 & 90.2778 & 95.2778 & 1.9215 \\
\hline
\end{tabular}

TABLE XLII

NN-BASED WEIGHTED PARALLEL-STRUCTURED CLASSIFIER USING 4 FEATURE POINTS OF NOISY TEST DATASET. NUMBER OF HIDDEN NODES: 15, TRANSFER FUNCTION OF HIDDEN NODES: HYPERBOLIC TANGENT SIGMOID TRANSFER FUNCTION.

\begin{tabular}{|c|c|c|c|c|}
\cline { 2 - 5 } \multicolumn{1}{c|}{} & \multicolumn{4}{c|}{ Recognition Accuracy (\%) } \\
\hline Material & Average & Min & Max & Std \\
\hline 1 & 90.0000 & 85.0000 & 100.0000 & 4.7140 \\
\hline 2 & 99.0000 & 95.0000 & 100.0000 & 2.1082 \\
\hline 3 & 98.5000 & 95.0000 & 100.0000 & 2.4152 \\
\hline 4 & 89.0000 & 75.0000 & 95.0000 & 6.5828 \\
\hline 5 & 99.5000 & 95.0000 & 100.0000 & 1.5811 \\
\hline 6 & 97.5000 & 95.0000 & 100.0000 & 2.6352 \\
\hline 7 & 100.0000 & 100.0000 & 100.0000 & 0.0000 \\
\hline 8 & 100.0000 & 100.0000 & 100.0000 & 0.0000 \\
\hline 9 & 100.0000 & 100.0000 & 100.0000 & 0.0000 \\
\hline 10 & 100.0000 & 100.0000 & 100.0000 & 0.0000 \\
\hline 11 & 100.0000 & 100.0000 & 100.0000 & 0.0000 \\
\hline 12 & 91.5000 & 70.0000 & 100.0000 & 8.8349 \\
\hline 13 & 100.0000 & 100.0000 & 100.0000 & 0.0000 \\
\hline 14 & 100.0000 & 100.0000 & 100.0000 & 0.0000 \\
\hline 15 & 73.5000 & 55.0000 & 85.0000 & 9.1439 \\
\hline 16 & 100.0000 & 100.0000 & 100.0000 & 0.0000 \\
\hline 17 & 100.0000 & 100.0000 & 100.0000 & 0.0000 \\
\hline 18 & 100.0000 & 100.0000 & 100.0000 & 0.0000 \\
\hline \hline Average & 96.5833 & 92.5000 & 98.8889 & 2.1120 \\
\hline
\end{tabular}

TABLE XLIII

NN-BASED WEIGHTED PARALLEL-STRUCTURED CLASSIFIER USING 5 FEATURE POINTS OF NOISY TEST DATASET. NUMBER OF HIDDEN NODES: 15, TRANSFER FUNCTION OF HIDDEN NODES: HYPERBOLIC TANGENT SIGMOID TRANSFER FUNCTION. 


\begin{tabular}{|c|c|c|c|c|}
\cline { 2 - 5 } \multicolumn{1}{c|}{} & \multicolumn{4}{c|}{ Recognition Accuracy (\%) } \\
\hline Material & Average & Min & Max & Std \\
\hline 1 & 100.0000 & 100.0000 & 100.0000 & 0.0000 \\
\hline 2 & 100.0000 & 100.0000 & 100.0000 & 0.0000 \\
\hline 3 & 89.5000 & 85.0000 & 95.0000 & 3.6893 \\
\hline 4 & 100.0000 & 100.0000 & 100.0000 & 0.0000 \\
\hline 5 & 100.0000 & 100.0000 & 100.0000 & 0.0000 \\
\hline 6 & 100.0000 & 100.0000 & 100.0000 & 0.0000 \\
\hline 7 & 100.0000 & 100.0000 & 100.0000 & 0.0000 \\
\hline 8 & 100.0000 & 100.0000 & 100.0000 & 0.0000 \\
\hline 9 & 100.0000 & 100.0000 & 100.0000 & 0.0000 \\
\hline 10 & 100.0000 & 100.0000 & 100.0000 & 0.0000 \\
\hline 11 & 95.0000 & 90.0000 & 100.0000 & 2.3570 \\
\hline 12 & 100.0000 & 100.0000 & 100.0000 & 0.0000 \\
\hline 13 & 100.0000 & 100.0000 & 100.0000 & 0.0000 \\
\hline 14 & 100.0000 & 100.0000 & 100.0000 & 0.0000 \\
\hline 15 & 80.5000 & 65.0000 & 90.0000 & 8.9598 \\
\hline 16 & 96.5000 & 95.0000 & 100.0000 & 2.4152 \\
\hline 17 & 100.0000 & 100.0000 & 100.0000 & 0.0000 \\
\hline 18 & 100.0000 & 100.0000 & 100.0000 & 0.0000 \\
\hline \hline Average & 97.8611 & 96.3889 & 99.1667 & 0.9679 \\
\hline
\end{tabular}

TABLE XLIV

NN-BASED TREE-STRUCTURED CLASSIFIER USING 3 FEATURE POINTS OF NOISY TEST DATASET. NUMBER OF HIDDEN NODES: 20, TRANSFER FUNCTION OF HIDDEN NODES: HYPERBOLIC TANGENT SIGMOID TRANSFER FUNCTION.

\begin{tabular}{|c|c|c|c|c|}
\cline { 2 - 5 } \multicolumn{1}{c|}{} & \multicolumn{4}{c|}{ Recognition Accuracy (\%) } \\
\hline Material & Average & Min & Max & Std \\
\hline 1 & 95.0000 & 90.0000 & 100.0000 & 2.3570 \\
\hline 2 & 95.5000 & 90.0000 & 100.0000 & 2.8382 \\
\hline 3 & 75.5000 & 65.0000 & 90.0000 & 6.8516 \\
\hline 4 & 100.0000 & 100.0000 & 100.0000 & 0.0000 \\
\hline 5 & 100.0000 & 100.0000 & 100.0000 & 0.0000 \\
\hline 6 & 100.0000 & 100.0000 & 100.0000 & 0.0000 \\
\hline 7 & 100.0000 & 100.0000 & 100.0000 & 0.0000 \\
\hline 8 & 100.0000 & 100.0000 & 100.0000 & 0.0000 \\
\hline 9 & 100.0000 & 100.0000 & 100.0000 & 0.0000 \\
\hline 10 & 100.0000 & 100.0000 & 100.0000 & 0.0000 \\
\hline 11 & 96.0000 & 90.0000 & 100.0000 & 3.1623 \\
\hline 12 & 99.5000 & 95.0000 & 100.0000 & 1.5811 \\
\hline 13 & 91.0000 & 90.0000 & 95.0000 & 2.1082 \\
\hline 14 & 100.0000 & 100.0000 & 100.0000 & 0.0000 \\
\hline 15 & 100.0000 & 100.0000 & 100.0000 & 0.0000 \\
\hline 16 & 100.0000 & 100.0000 & 100.0000 & 0.0000 \\
\hline 17 & 100.0000 & 100.0000 & 100.0000 & 0.0000 \\
\hline 18 & 100.0000 & 100.0000 & 100.0000 & 0.0000 \\
\hline \hline Average & 97.3611 & 95.5556 & 99.1667 & 1.0499 \\
\hline
\end{tabular}

TABLE XLV

NN-BASED TREE-STRUCTURED CLASSIFIER USING 4 FEATURE POINTS OF NOISY TEST DATASET. NUMBER OF HIDDEN NODES: 20, TRANSFER FUNCTION OF HIDDEN NODES: HYPERBOLIC TANGENT SIGMOID TRANSFER FUNCTION. 


\begin{tabular}{|c|c|c|c|c|}
\cline { 2 - 5 } \multicolumn{1}{c|}{} & \multicolumn{4}{c|}{ Recognition Accuracy (\%) } \\
\hline Material & Average & Min & Max & Std \\
\hline 1 & 100.0000 & 100.0000 & 100.0000 & 0.0000 \\
\hline 2 & 96.0000 & 85.0000 & 100.0000 & 5.1640 \\
\hline 3 & 100.0000 & 100.0000 & 100.0000 & 0.0000 \\
\hline 4 & 100.0000 & 100.0000 & 100.0000 & 0.0000 \\
\hline 5 & 100.0000 & 100.0000 & 100.0000 & 0.0000 \\
\hline 6 & 100.0000 & 100.0000 & 100.0000 & 0.0000 \\
\hline 7 & 100.0000 & 100.0000 & 100.0000 & 0.0000 \\
\hline 8 & 100.0000 & 100.0000 & 100.0000 & 0.0000 \\
\hline 9 & 100.0000 & 100.0000 & 100.0000 & 0.0000 \\
\hline 10 & 100.0000 & 100.0000 & 100.0000 & 0.0000 \\
\hline 11 & 100.0000 & 100.0000 & 100.0000 & 0.0000 \\
\hline 12 & 100.0000 & 100.0000 & 100.0000 & 0.0000 \\
\hline 13 & 100.0000 & 100.0000 & 100.0000 & 0.0000 \\
\hline 14 & 100.0000 & 100.0000 & 100.0000 & 0.0000 \\
\hline 15 & 100.0000 & 100.0000 & 100.0000 & 0.0000 \\
\hline 16 & 100.0000 & 100.0000 & 100.0000 & 0.0000 \\
\hline 17 & 100.0000 & 100.0000 & 100.0000 & 0.0000 \\
\hline 18 & 100.0000 & 100.0000 & 100.0000 & 0.0000 \\
\hline \hline Average & 99.7778 & 99.1667 & 100.0000 & 0.2869 \\
\hline
\end{tabular}

TABLE XLVI

NN-BASED TREE-STRUCTURED CLASSIFIER USING 5 FEATURE POINTS OF NOISY TEST DATASET. NUMBER OF HIDDEN NODES: 20, TRANSFER FUNCTION OF HIDDEN NODES: HYPERBOLIC TANGENT SIGMOID TRANSFER FUNCTION.

\begin{tabular}{|c|c|c|c|c|}
\cline { 2 - 5 } \multicolumn{1}{c|}{} & \multicolumn{4}{c|}{ Recognition Accuracy (\%) } \\
\hline Material & Average & Min & Max & Std \\
\hline 1 & 93.0000 & 90.0000 & 100.0000 & 4.2164 \\
\hline 2 & 88.5000 & 75.0000 & 100.0000 & 8.8349 \\
\hline 3 & 93.0000 & 85.0000 & 100.0000 & 4.8305 \\
\hline 4 & 92.0000 & 85.0000 & 100.0000 & 4.2164 \\
\hline 5 & 95.0000 & 90.0000 & 100.0000 & 3.3333 \\
\hline 6 & 100.0000 & 100.0000 & 100.0000 & 0.0000 \\
\hline 7 & 100.0000 & 100.0000 & 100.0000 & 0.0000 \\
\hline 8 & 100.0000 & 100.0000 & 100.0000 & 0.0000 \\
\hline 9 & 94.5000 & 90.0000 & 100.0000 & 2.8382 \\
\hline 10 & 98.5000 & 95.0000 & 100.0000 & 2.4152 \\
\hline 11 & 84.0000 & 75.0000 & 90.0000 & 5.1640 \\
\hline 12 & 91.0000 & 80.0000 & 100.0000 & 6.1464 \\
\hline 13 & 100.0000 & 100.0000 & 100.0000 & 0.0000 \\
\hline 14 & 100.0000 & 100.0000 & 100.0000 & 0.0000 \\
\hline 15 & 91.0000 & 75.0000 & 100.0000 & 7.3786 \\
\hline 16 & 92.5000 & 90.0000 & 95.0000 & 2.6352 \\
\hline 17 & 84.5000 & 75.0000 & 90.0000 & 4.9721 \\
\hline 18 & 92.5000 & 90.0000 & 95.0000 & 2.6352 \\
\hline \hline Average & 93.8889 & 88.6111 & 98.3333 & 3.3120 \\
\hline
\end{tabular}

TABLE XLVII

K-NEAREST NEIGHBOR CLASSIFIER USING 3 FEATURE POINTS OF NOISY TEST DATASET. 


\begin{tabular}{|c|c|c|c|c|}
\cline { 2 - 5 } \multicolumn{1}{c|}{} & \multicolumn{4}{c|}{ Recognition Accuracy (\%) } \\
\hline Material & Average & Min & Max & Std \\
\hline 1 & 86.6667 & 80.0000 & 90.0000 & 5.7735 \\
\hline 2 & 71.6667 & 65.0000 & 80.0000 & 7.6376 \\
\hline 3 & 66.6667 & 55.0000 & 80.0000 & 12.5831 \\
\hline 4 & 90.0000 & 90.0000 & 90.0000 & 0.0000 \\
\hline 5 & 91.6667 & 85.0000 & 100.0000 & 7.6376 \\
\hline 6 & 96.6667 & 95.0000 & 100.0000 & 2.8868 \\
\hline 7 & 100.0000 & 100.0000 & 100.0000 & 0.0000 \\
\hline 8 & 100.0000 & 100.0000 & 100.0000 & 0.0000 \\
\hline 9 & 96.6667 & 95.0000 & 100.0000 & 2.8868 \\
\hline 10 & 90.0000 & 85.0000 & 100.0000 & 8.6603 \\
\hline 11 & 88.3333 & 85.0000 & 90.0000 & 2.8868 \\
\hline 12 & 81.6667 & 75.0000 & 90.0000 & 7.6376 \\
\hline 13 & 95.0000 & 95.0000 & 95.0000 & 0.0000 \\
\hline 14 & 96.6667 & 95.0000 & 100.0000 & 2.8868 \\
\hline 15 & 75.0000 & 65.0000 & 85.0000 & 10.0000 \\
\hline 16 & 76.6667 & 75.0000 & 80.0000 & 2.8868 \\
\hline 17 & 75.0000 & 70.0000 & 80.0000 & 5.0000 \\
\hline 18 & 98.3333 & 95.0000 & 100.0000 & 2.8868 \\
\hline \hline Average & 87.5926 & 83.6111 & 92.2222 & 4.5695 \\
\hline
\end{tabular}

TABLE XLVIII

K-NEAREST NEIGHBOR CLASSIFIER USING 4 FEATURE POINTS OF NOISY TEST DATASET.

\begin{tabular}{|c|c|c|c|c|}
\cline { 2 - 5 } \multicolumn{1}{c|}{} & \multicolumn{4}{c|}{ Recognition Accuracy (\%) } \\
\hline Material & Average & Min & Max & Std \\
\hline 1 & 96.5000 & 90.0000 & 100.0000 & 3.3747 \\
\hline 2 & 92.5000 & 90.0000 & 95.0000 & 2.6352 \\
\hline 3 & 99.5000 & 95.0000 & 100.0000 & 1.5811 \\
\hline 4 & 93.0000 & 90.0000 & 100.0000 & 3.4960 \\
\hline 5 & 100.0000 & 100.0000 & 100.0000 & 0.0000 \\
\hline 6 & 100.0000 & 100.0000 & 100.0000 & 0.0000 \\
\hline 7 & 100.0000 & 100.0000 & 100.0000 & 0.0000 \\
\hline 8 & 71.5000 & 65.0000 & 75.0000 & 3.3747 \\
\hline 9 & 100.0000 & 100.0000 & 100.0000 & 0.0000 \\
\hline 10 & 100.0000 & 100.0000 & 100.0000 & 0.0000 \\
\hline 11 & 97.5000 & 90.0000 & 100.0000 & 3.5355 \\
\hline 12 & 94.5000 & 85.0000 & 100.0000 & 4.3780 \\
\hline 13 & 81.5000 & 75.0000 & 90.0000 & 4.7434 \\
\hline 14 & 100.0000 & 100.0000 & 100.0000 & 0.0000 \\
\hline 15 & 90.5000 & 75.0000 & 100.0000 & 8.3166 \\
\hline 16 & 66.0000 & 50.0000 & 75.0000 & 8.0966 \\
\hline 17 & 39.5000 & 35.0000 & 50.0000 & 4.3780 \\
\hline 18 & 73.0000 & 60.0000 & 85.0000 & 7.1492 \\
\hline \hline Average & 88.6389 & 83.3333 & 92.7778 & 3.0588 \\
\hline
\end{tabular}

TABLE XLIX

K-NEAREST NEIGHBOR CLASSIFIER USING 5 FEATURE POINTS OF NOISY TEST DATASET. 


\begin{tabular}{|c|c|c|c|c|}
\cline { 2 - 5 } \multicolumn{1}{c|}{} & \multicolumn{4}{c|}{ Recognition Accuracy (\%) } \\
\hline Material & Average & Min & Max & Std \\
\hline 1 & 100.0000 & 100.0000 & 100.0000 & 0.0000 \\
\hline 2 & 99.5000 & 95.0000 & 100.0000 & 1.5811 \\
\hline 3 & 86.0000 & 80.0000 & 90.0000 & 3.1623 \\
\hline 4 & 100.0000 & 100.0000 & 100.0000 & 0.0000 \\
\hline 5 & 100.0000 & 100.0000 & 100.0000 & 0.0000 \\
\hline 6 & 100.0000 & 100.0000 & 100.0000 & 0.0000 \\
\hline 7 & 100.0000 & 100.0000 & 100.0000 & 0.0000 \\
\hline 8 & 100.0000 & 100.0000 & 100.0000 & 0.0000 \\
\hline 9 & 98.0000 & 95.0000 & 100.0000 & 2.5820 \\
\hline 10 & 100.0000 & 100.0000 & 100.0000 & 0.0000 \\
\hline 11 & 99.5000 & 95.0000 & 100.0000 & 1.5811 \\
\hline 12 & 100.0000 & 100.0000 & 100.0000 & 0.0000 \\
\hline 13 & 100.0000 & 100.0000 & 100.0000 & 0.0000 \\
\hline 14 & 100.0000 & 100.0000 & 100.0000 & 0.0000 \\
\hline 15 & 0.5000 & 0.0000 & 5.0000 & 1.5811 \\
\hline 16 & 100.0000 & 100.0000 & 100.0000 & 0.0000 \\
\hline 17 & 100.0000 & 100.0000 & 100.0000 & 0.0000 \\
\hline 18 & 100.0000 & 100.0000 & 100.0000 & 0.0000 \\
\hline \hline Average & 93.5278 & 92.5000 & 94.1667 & 0.5826 \\
\hline
\end{tabular}

TABLE L

NAIVE BAYES CLASSIFIER USING 3 FEATURE POINTS OF NOISY TEST DATASET.

\begin{tabular}{|c|c|c|c|c|}
\cline { 2 - 5 } \multicolumn{1}{c|}{} & \multicolumn{4}{c|}{ Recognition Accuracy (\%) } \\
\hline Material & Average & Min & Max & Std \\
\hline 1 & 100.0000 & 100.0000 & 100.0000 & 0.0000 \\
\hline 2 & 97.0000 & 95.0000 & 100.0000 & 2.5820 \\
\hline 3 & 77.0000 & 65.0000 & 90.0000 & 8.2327 \\
\hline 4 & 100.0000 & 100.0000 & 100.0000 & 0.0000 \\
\hline 5 & 100.0000 & 100.0000 & 100.0000 & 0.0000 \\
\hline 6 & 100.0000 & 100.0000 & 100.0000 & 0.0000 \\
\hline 7 & 100.0000 & 100.0000 & 100.0000 & 0.0000 \\
\hline 8 & 100.0000 & 100.0000 & 100.0000 & 0.0000 \\
\hline 9 & 100.0000 & 100.0000 & 100.0000 & 0.0000 \\
\hline 10 & 100.0000 & 100.0000 & 100.0000 & 0.0000 \\
\hline 11 & 100.0000 & 100.0000 & 100.0000 & 0.0000 \\
\hline 12 & 100.0000 & 100.0000 & 100.0000 & 0.0000 \\
\hline 13 & 100.0000 & 100.0000 & 100.0000 & 0.0000 \\
\hline 14 & 100.0000 & 100.0000 & 100.0000 & 0.0000 \\
\hline 15 & 0.5000 & 0.0000 & 5.0000 & 1.5811 \\
\hline 16 & 100.0000 & 100.0000 & 100.0000 & 0.0000 \\
\hline 17 & 100.0000 & 100.0000 & 100.0000 & 0.0000 \\
\hline 18 & 100.0000 & 100.0000 & 100.0000 & 0.0000 \\
\hline \hline Average & 93.0278 & 92.2222 & 94.1667 & 0.6887 \\
\hline
\end{tabular}

TABLE LI

NAIVE BAYES CLASSIFIER USING 4 FEATURE POINTS OF NOISY TEST DATASET. 


\begin{tabular}{|c|c|c|c|c|}
\cline { 2 - 5 } \multicolumn{1}{c|}{} & \multicolumn{4}{c|}{ Recognition Accuracy (\%) } \\
\hline Material & Average & Min & Max & Std \\
\hline 1 & 100.0000 & 100.0000 & 100.0000 & 0.0000 \\
\hline 2 & 100.0000 & 100.0000 & 100.0000 & 0.0000 \\
\hline 3 & 90.0000 & 80.0000 & 95.0000 & 5.7735 \\
\hline 4 & 100.0000 & 100.0000 & 100.0000 & 0.0000 \\
\hline 5 & 100.0000 & 100.0000 & 100.0000 & 0.0000 \\
\hline 6 & 100.0000 & 100.0000 & 100.0000 & 0.0000 \\
\hline 7 & 100.0000 & 100.0000 & 100.0000 & 0.0000 \\
\hline 8 & 100.0000 & 100.0000 & 100.0000 & 0.0000 \\
\hline 9 & 100.0000 & 100.0000 & 100.0000 & 0.0000 \\
\hline 10 & 100.0000 & 100.0000 & 100.0000 & 0.0000 \\
\hline 11 & 100.0000 & 100.0000 & 100.0000 & 0.0000 \\
\hline 12 & 100.0000 & 100.0000 & 100.0000 & 0.0000 \\
\hline 13 & 100.0000 & 100.0000 & 100.0000 & 0.0000 \\
\hline 14 & 100.0000 & 100.0000 & 100.0000 & 0.0000 \\
\hline 15 & 0.5000 & 0.0000 & 5.0000 & 1.5811 \\
\hline 16 & 100.0000 & 100.0000 & 100.0000 & 0.0000 \\
\hline 17 & 100.0000 & 100.0000 & 100.0000 & 0.0000 \\
\hline 18 & 100.0000 & 100.0000 & 100.0000 & 0.0000 \\
\hline \hline Average & 93.9167 & 93.3333 & 94.4444 & 0.4086 \\
\hline
\end{tabular}

TABLE LII

NAIVE BAYES CLASSIFIER USING 5 FEATURE POINTS OF NOISY TEST DATASET. 


\section{REFERENCES}

[1] J. Zurada, "Could decision trees improve the classification accuracy and interpretability of loan granting decisions?" in Proceedings of the 43rd Hawaii International Conference on System Sciences (HICSS), Jan. 2010, pp. 1-9.

[2] J. Pradeep, E. Srinivasan, and S. Himavathi, "Neural network based handwritten character recognition system without feature extraction," in Proceedings of the International Conference on Computer, Communication and Electrical Technology (ICCCET), Jan. 2011, pp. 40-44.

[3] J.-T. Chien, "Linear regression based bayesian predictive classification for speech recognition," IEEE Transactions on Speech and Audio Processing, vol. 11, no. 1, pp. 70-79, Jan. 2003.

[4] G. L. Marcialis and F. Roli, "Fusion of multiple fingerprint matchers by single-layer perceptron with class-separation loss function," Pattern Recognition Letters, vol. 26, no. 12, pp. 1830-1839, Sep. 2005.

[5] G. P. Zhang, "Neural networks for classification: a survey," IEEE Transactions on Systems, Man, and Cybernetics, Part C: Applications and Reviews, vol. 30, no. 4, pp. 451-462, Nov. 2000.

[6] A. Tellaeche, X. P. Burgos-Artizzu, G. Pajares, and A. Ribeiro, "A vision-based method for weeds identification through the Bayesian decision theory," Pattern Recognition, vol. 41, no. 2, pp. 521-530, Feb 2008.

[7] M.-L. Zhang and Z.-H. Zhou, "A k-nearest neighbor based algorithm for multi-label classification," in Proceedings of the IEEE International Conference on Granular Computing, vol. 2, 2005, pp. 718-721.

[8] H. Zhang, A. C. Berg, M. Maire, and J. Malik, "SVM-KNN: Discriminative nearest neighbor classification for visual category recognition," in Proceedings of the IEEE Computer Society Conference on Computer Vision and Pattern Recognition, vol. 2, 2006, pp. 2126-2136.

[9] C. J. C. Burges, "A tutorial on support vector machines for pattern recognition," Data mining and knowledge discovery, vol. 2, no. 2, pp. 121-167, 1998.

[10] S. Kotsiantis, "Supervised machine learning: A review of classification techniques," Informatica, vol. 31, pp. 249-268, Jul. 2007.

[11] Y.-S. Chen and T.-H. Chu, "A neural network classification tree," in Proceedings of the IEEE International Conference on Neural Networks, vol. 1, Dec. 1995, pp. 409-413.

[12] A. Santillana Fernandez, C. Delgado-Mata, and R. Velazquez, "Training a single-layer perceptron for an approximate edge detection on a digital image," in Proceedings of the International Conference on Technologies and Applications of Artificial Intelligence (TAAI), Nov. 2011, pp. 189-193.

[13] U. Kumar, S. K. Raja, C. Mukhopadhyay, and T. Ramachandra, "Hybrid bayesian classifier for improved classification accuracy," IEEE Geoscience and Remote Sensing Letters, vol. 8, no. 3, pp. 474-477, Nov. 2011.

[14] L. Zhou, L. Wang, X. Ge, and Q. Shi, "A clustering-based knn improved algorithm CLKNN for text classification,” in Proceedings of the $2^{\text {nd }}$ International Asia Conference on Informatics in Control, Automation and Robotics (CAR), vol. 3, 2010, pp. $212-215$.

[15] K. Hornik, M. Stinchcombe, and H. White, "Universal approximation of an unknown mapping and its derivatives using multilayer feedforward networks," Neural networks, vol. 3, no. 5, pp. 551-560, 1990.

[16] K. Schierholt and C. H. Dagli, "Stock market prediction using different neural network classification architectures," in Proceedings of the IEEEIAFE 1996 Conference on Computational Intelligence for Financial Engineering, 1996, pp. 72-78.

[17] X. Li, J. Cervantes, and W. Yu, "A novel SVM classification method for large data sets," in IEEE International Conference on Granular Computing (GrC), 2010, pp. 297-302. 
[18] A. M. Okamura, "Methods for haptic feedback in teleoperated robot-assisted surgery," Industrial Robot: An International Journal, vol. 31, no. 6, pp. 499-508, 2004.

[19] O. A. J. Van der Meijden and M. P. Schijven, "The value of haptic feedback in conventional and robot-assisted minimal invasive surgery and virtual reality training: a current review," Surgical endoscopy, vol. 23, no. 6, pp. 1180-1190, 2009.

[20] C.-H. King, M. O. Culjat, M. L. Franco, C. E. Lewis, E. P. Dutson, W. S. Grundfest, and J. W. Bisley, "Tactile feedback induces reduced grasping force in robot-assisted surgery," Haptics, IEEE Transactions on, vol. 2, no. 2, pp. 103-110, 2009.

[21] G. I. Barbash and S. A. Glied, "New technology and health care costs-the case of robot-assisted surgery," New England Journal of Medicine, vol. 363, no. 8, pp. 701-704, 2010.

[22] R. Ozawa, J.-H. Bae, and S. Arimoto, "Multi-fingered dynamic blind grasping with tactile feedback in a horizontal plane," in Proceedings of the 2006 IEEE International Conference on Robotics and Automation (ICRA), 2006, pp. 1006-1011.

[23] H. Dang, J. Weisz, and P. K. Allen, "Blind grasping: Stable robotic grasping using tactile feedback and hand kinematics," in Proceedings of the 2011 IEEE International Conference on Robotics and Automation (ICRA), 2011, pp. 5917-5922.

[24] H. Liu, X. Song, T. Nanayakkara, L. D. Seneviratne, and K. Althoefer, "A computationally fast algorithm for local contact shape and pose classification using a tactile array sensor," in Proceedings of 2012 IEEE International Conference on Robotics and Automation (ICRA). IEEE, 2012, pp. 1410-1415.

[25] R. E. Fan, M. O. Culjat, C.-H. King, M. L. Franco, R. Boryk, J. W. Bisley, E. Dutson, and W. S. Grundfest, "A haptic feedback system for lower-limb prostheses," IEEE Transactions on Neural Systems and Rehabilitation Engineering, vol. 16, no. 3, pp. 270-277, 2008.

[26] N. Jamali, "Material classification by tactile sensing using surface textures," in Proceedings of the 2007 IEEE International Conference on Image Processing, May 2010, pp. 2336-2341.

[27] V. Thilak, "Material classification using passive polametric imagery," in Proceedings of the 2007 IEEE International Conference on Image Processing, Sept. 2007, pp. 121-124.

[28] N. Charniya, "Neural network based sensor for classification of material type and its surface properties," in Proceedings of the 2007 IEEE International Joint Conference on Neural Networks, Aug. 2007, pp. 424-429.

[29] J. E. ODoherty, M. A. Lebedev, P. J. Ifft, K. Z. Zhuang, S. Shokur, H. Bleuler, and M. A. L. Nicolelis, "Active tactile exploration using a brain-machine-brain interface," Nature, vol. 479, no. 7372, pp. 228-231, 2011.

[30] X. Song, H. Liu, J. Bimbo, K. Althoefer, and L. D. Seneviratne, "A novel dynamic slip prediction and compensation approach based on haptic surface exploration," in Proceedings of 2012 IEEE/RSJ International Conference on Intelligent Robots and Systems (IROS),. IEEE, 2012, pp. 4511-4516.

[31] X. Song, H. Liu, K. Althoefer, T. Nanayakkara, and L. D. Seneviratne, "Efficient break-away friction ratio and slip prediction based on haptic surface exploration," IEEE Transactions on Robotics, 2013, DOI: 10.1109/TRO.2013.2279630.

[32] K. F. Leung, F. H. F. Leung, H. K. Lam, and S. H. Ling, "On interpretation of graffiti digits and characters for eBooks: neural-fuzzy network and genetic algorithm approach,” IEEE Trans. on Industrial Electronics, vol. 51, no. 2, pp. 464-471, Apr. 2004.

[33] H. K. Lam and F. H. F. Leung, "Digit and command interpretation for electronic book using neural network and genetic algorithm," IEEE Trans. on Systems, Man, and Cybernetics, Part B: Cybernetics, vol. 34, no. 6, pp. 2273-2283, Dec. 2004.

[34] H. K. Lam and J. Prada, "Interpretation of handwritten single-stroke graffiti using support vector machines," International Journal of Computational Intelligence and Applications, vol. 8, no. 04, pp. 369-393, Dec. 2009.

[35] H. Liu, X. Song, J. Bimbo, L. Seneviratne, and K. Althoefer, "Surface material recognition through haptic exploration using an 
intelligent contact sensing finger," in Proceedings of the 2012 IEEE/RSJ International Conference on Intelligent Robots and Systems (IROS). IEEE, 2012, pp. 52-57. 